\title{
Updated Checklist of Food Plants of Macrosiphini (Aphididae: Hemiptera) in India-2
}

\author{
${ }^{1}$ Garima Singh, ${ }^{2}$ Rajendra Singh \\ ${ }^{1}$ Department of Zoology, Rajasthan University, Jaipur, Rajasthan. \\ ${ }^{2}$ Department of Zoology, D.D.U. Gorakhpur University, Gorakhpur, U.P.
}

\begin{abstract}
The Macrosiphini is one of the 2 tribes of the subfamily Aphidinae (Aphididae: Hemiptera) containing about 2166_species/subspecies assigned to 242 genera. Out of these, only 96 genera and 374 species are recorded from India infesting hundreds of plant species belonging to several families. The food plants of Indian Macrosiphini is catalogued alphabetically (aphid species-wise) in three parts. In this contribution, food plants of 38 genera_and 148 species of aphids belonging to the tribe Macrosiphini (Elatobium to Myzus) are catalogued. These aphids feeds on 416 plant species belonging to 98 families. Most suffered families are Asteraceae (62 species) followed by Rosaceae (41 species), Brassicaceae (26 species), Lamiaceae (23 species), Fabaceae (18 species), Pteridaceae (14 species), and Solanaceae (13 species). Among them, several species are crops of agricultural and horticultural importance. The update checklist of food plants of rest of Macrosiphini will be dealt in successive article.
\end{abstract}

Keywords: Food plant catalogue, Macrosiphini, aphid, checklist.

\section{INTRODUCTION}

The tribe Macrosiphini Wilson, 1910 is one of the two tribes (Aphidini and Macrosiphini) of the subfamily Aphidinae (Aphididae: Hemiptera) which is the largest subfamily of the aphids containing 2166 species assigned to 242 genera [1]. Several members of the tribe are major agricultural pests damaging the crops directly by sucking their sap or by transmitting viral diseases. Myzus persicae (Sulzer) alone transmit about 180 plant viruses [2,3]. In the most of the literature published earlier, several errors crept in scientific names of the aphids and plants even in the recent ones. It happens because such contents become outdated quickly and, due to their perceived comprehensiveness, readers sometimes overlook newer sources of data [4]. Additionally, the researches on aphid taxonomy as well as their host plants is continued with the description of new taxa, the modified status of others, and the publication of other nomenclatural decisions. In the present compilation, attempts have been made to correct these errors in the scientific names of the aphids following recent taxonomic literatures $[1,5,6,7,8]$ and for plants, http://www.ars-grin.gov and http://www.the plantlist.org (version 1.1). At several places, their synonymies were also mentioned.

\section{APHID - FoOd Plants Record of MaCROSIPHINI}

The food plants of Aphidini [9] and first part of Macrosiphini [10] were recently catalogued. The present paper deals with the second part of Macrosiphini and their food plants in India. Aphid species marked with $\S$ seems to be invalid while species marked with * are wrongly reported for another valid species. Following is their food plant record:

1. Elatobium sclerotica Agarwala, Pramanik \& Raychaudhuri, 1982

- Unidentified plant [11]

\section{Elatobium sp.}

- Eurya sp. (Ternstroemiaceae) [12]

3. Ericolophium dubium (Chakrabarti \& Maity, 1984)

= Neoacyrthosiphon dubium Chakrabarti \& Maity, 1984

- Populus ciliata Wall. ex Royle (Salicaceae) [13]

- Salix denticulata Andersson (Salicaceae) [13, 14] 
- Salix elaeagnos Scop. (=Salix elegans auct. non.) (Salicaceae) [13]

- Salix tetrasperma Roxb. (Salicaceae) [15]

- Salix sp. (Salicaceae) [13, 14, 16]

4. Ericolophium euryae (Takahashi, 1937)

= Metopolophium (Metopolophium) euryae (Takahashi, 1937) [17]

= Acyrthosiphon (Metopolophium) euryae (Takahashi, 1937) [18]

- Eurya acuminata L. (Ternstroemiaceae) [19]

- Eurya japonica Thunb. (Ternstroemiaceae) [18, 20]

5. Ericolophium holsti (Takahashi, 1935)

= Neoacyrthosiphon holsti (Takahashi, 1935)

= Neoacyrthosiphon (Pseudacyrthosiphon) holsti (Takahashi, 1935)

= Pseudoacyrthosiphon (Pseudoacrythosiphon)holsti (Takahashi, 1935) [17]

= Neomasonaphis rhododendris Chakrabarti, Mandal \& Raha, 1983

- Rhododendron arboreum Sm. (Ericaceae) [20-23]

- Rhododendron sp. (Ericaceae) [13, 20, 21, 24- 29]

6. Ericolophium rhododendri (Ghosh, Ghosh \& Raychaudhuri, 1971)

= Neoacyrthosiphon rhododendri Ghosh, Ghosh \& Raychaudhuri, 1970 (1971)

- Leucosceptrum cannum Sm. (Lamiaceae) [30]

- Rhododendron arboreum Sm. (Ericaceae) [20, 22, 31]

- Rhododendron sp. (Ericaceae) [24]

7. Ericolophium sikkimensis Agarwala \& Mahapatra, 1986

- Undet.: Ericaceae [32]

8. Ericolophium taiheisanum ovalifolii (Ghosh, Ghosh \& Raychaudhuri, 1971)

= Neoacyrthosiphon taiheisanum ovalifolii Ghosh, Ghosh \& Raychaudhuri, 1970 (1971)

- Lyonia ovalifolia (Wall.) Drude (=Pieris ovalifolis (Wall. D. Don)) (Ericaceae) [20, 31]

- Rhododendron sp. (Ericaceae) [22,33]

9. Ericolophium ? taiheisanum (Takahashi, 1935)

= Neoacyrthosiphon ? taiheisanum (Takahashi, 1935)

- Rhododendron sp. (Ericaceae) [34]

10. Ericolophium takahashii (Ghosh, 1969)

= Neoacyrthosiphon takahashii Ghosh, 1969

= Pseudoacyrthosiphon (Anacyrthosiphon) takahashii (Ghosh, 1969) [17]

= Neoacyrthosiphon (Pseudacyrthosiphon) nepalensis Ghosh, Basu \& Raychaudhuri, 1973

= Neoacyrthosiphon (Pseudacyrthosiphon) takahashii Ghosh, 1969 [ 35, 36, 37]

- Anemone rivularis DC. (Ranunculaceae) [37]

- Rhododendron arboreum Sm. (Ericaceae) [20, 23]

- Rhododendron campestris ? (Ericaceae) [13]

- Rhododendron campylocarpum Hook.f. (Ericaceae) [23, 36, 37, 38-41]

- Rhododendron sp. (Ericaceae) [23]

11.Eucarazzia elegans (Ferrari, 1872)

= Rhopalosiphoninus sensoriatus Chakrabarti, 1978

- Unidentified plants [43]

12. Ericolophium sp.

- Unidentified plant [44]

13. Eumyzus darjeelingensis Basu \& Raychaudhuri, 1974

- Hydrangea paniculata Siebold (Hydrangeaceae) [20, 45]

- Hydrangea sp. (Hydrangeaceae) [46]

- Prunus cornuta (Wall. ex Royle) Steud. (Rosaceae) [13] 
14. Eumyzus eastopi Maity \& Chakrabarti, 1982

- Prunus sp. (Rosaceae) [12]

- Sorbus vestita (Wall. ex G. Don) Lodd. (=Pyrus vestita Wall ex G. Don) (Rosaceae) [47, 48]

15. Eumyzus himalaya Agarwala, Pramanik \& Raychaudhuri, 1982

- Indet.: Cucurbitaceae [11]

16. Eumyzus hydrangi Chakrabarti \& Bhattacharya, 1985

- Hydrangea scandens (L.f.) Ser. (Hydrangeaceae) [47]

- Hydrangea sp. (Hydrangeaceae) [49, 50]

17. Eumyzus impatiensae (Shinji, 1924)

- Impatiens sp. (Balsaminaceae) [12, 47, 51]

18. Eumyzus indicus Medda \& Chakrabarti, 1992

- Impatiens glandulifera Royle (=Impatiens roylei Walp.) (Balsaminaceae) [52]

- Impatiens scabrida DC. (Balsaminaceae) [13, 52]

- Impatiens sp. (Balsaminaceae) [52]

19. Eumyzus nokuli Raychaudhuri, Singh \& Raychaudhuri, 1984

- Senecio sp. (Asteraceae) [53]

20. Eumyzus pruni Chakrabarti \& Bhattacharya, 1985

- Prunus cornuta (Wall. ex Royle) Steud. (Rosaceae) [47, 54, 55]

21. Eumyzus prunicolus Medda \& Chakrabarti, 1986

- Prunus padus L. (Rosaceae) $[54,56]$

22. Eumyzus simlaensis Bhattacharya, 1994

- Prunus padus L. (Rosaceae) [13]

- Prunus sp. (Rosaceae) [57]

23. Eumyzus sp.

- Nepeta cataria L. (Lamiaceae) [58]

24. Hayhurstia atriplicis atriplicis (Linnaeus, 1761)

= Hyalopterus carii Theobald, 1929 [59]

= Siphocoryne coriandri Das [59]

= Hyalopterus atriplicis (Linnaeus) [60, 61]

- Anacardium occidentale L. (Anacardiaceae) [62]

- Chenopodium album L. (Chenopodiaceae) [20, 37, 39, 59, 61, 63- 67]

- Chenopodium sp. (Chenopodiaceae ) [20, 59, 60, 68-72]

- Leycesteria formosa Wall. (Caprifoliaceae) [13]

- Prunus dulcis (Mill.) D.A. Webb. (=Prunus amygdalus Batsc.) (Rosaceae) [63]

- Pyrus pashia Buch.-Ham. ex D. Don (Rosaceae) [63]

- Sonchus sp. (Asteraceae) [61]

25. Hillerislambersia darjeelingi Basu, 1967

- Holboellia latifolia Wall. (Lardizabalaceae) [20]

- Lonicera sp. (Caprifoliaceae) [20, 22, 68]

- Rhododendron sp. (Ericaceae) [20]

26. Himalayaphis anemones Ghosh \& Verma, 1973

- Anemone sp. (Ranunculaceae) [71,73]

27. Hyadaphis coriandri (Das, 1918)

= Brevicoryne coriandri Das, 1918 [74]

= Hyadaphis corianderi (Das, 1918) [75]

= Hyalopterus carii Theobald, 1929 [59, 76, 77]

- Ammi majus L. (Apiaceae) [62]

- Anethum graveolens L. (Apiaceae) [78]

- Anethum graveolens L. (=Peucedanum graveolens (L.) (Apiaceae) [59, 66] 
- Atriplex hortensis L. (Chenopodiaceae) [67]

- Carum carvi L. (Apiaceae) [59]

- Coriandrum sativum L. (Apiaceae) [20, 22, 39, 59, 66, 67, 79-84]

- Coriandrum sp. (Apiaceae) $[69,85,86]$

- Cuminum sativum L. (Apiaceae) [59, 87]

- Daucus carota L. (Apiaceae) [59, 83]

- Foeniculum vulgare Mill. (Apiaceae) [39, 59, 79, 87]

- Helianthus annuus L. (Asteraceae) [39]

- Rubus plicatus Weihe \& Nees (=Rubus fruticosus L.) (Rosaceae) [26]

- Trachyspermum ammi (L.) Sprg. ex Turvil (=Carum copticum (L.) C. B. Clarke) (Apiaceae) [59, $62,76,77,87,88]$

- Trifolium alexandrinum L. (Fabaceae) [89, 90]

28. Hyadaphis foeniculi foeniculi (Passerini, 1860)

- Heracleum canadicans Wall. ex DC. (Apiaceae) $[39,91]$

29. Hyalomyzus fragaricola Ghosh, 1986

- Fragaria sp. (Rosaceae) [85, 92]

30. Hyalomyzus himachali Bhattacharya, 1994

- Rubus sp. (Rosaceae) [92]

31. Hyalomyzus raoi Hille Ris Lambers, 1973

= Neohyalomyzus raoi (Hille Ris Lambers, 1973) [17, 36]

- Artemisia sp. (Asteraceae) [20, 22]

- Chenopodium sp. (Chenopodiaceae ) [20, 22]

- Helicteres sp. (Malvaceae (=Sterculiaceae)) [20, 22, 93]

- Oplismenus compositus (L.) P. Beauv. (Poaceae) [22]

- Rosa sp. (Rosaceae) [94]

- Rubus ellipticus Sm. (Rosaceae) [12, 13, 22, 26, 29, 67, 92, 94, 95]

- Rubus moluccanus L. (=Rubus mollucata auct.) (Rosaceae) [96]

- Rubus paniculatus Sm. (Rosaceae) [35, 39]

- Rubus reticulatus A. Kern. (Rosaceae) [20]

- Rubus sp. (Rosaceae) [97]

32. Hyalomyzus sensoriatus (Mason, 1940)

- Unidentified plants [20, 36, 94]

33. Hyalomyzus sp.

- Peucedanum tenuifolium Thunb. (Apiaceae) [71]

- Rubus sp. (Rosaceae) [64]

34. Hydronaphis colocasiae Raychaudhuri, Raha \& Raychaudhuri, 1977

- Colocasia sp. (Araceae) [98-100]

35. Hyperomyzus (Hyperomyzus) carduellinus (Theobald, 1915)

= Hyperomyzus oleraceae (van der Goot, 1917) [59, 101-103]

- Ageratum sp. (Asteraceae) [20, 22]

- Datura metel L. (=Datura fastuosa L.) (Solanaceae)83

- Echinops sp. (Asteraceae) [20, 22]

- Emilia sonchifolia L. (Asteraceae) [20, 22, 26, 28, 104, 105]

- Gynura cusimbua (D. Don) S. Moore (=Gynura angulosa DC.) (Asteraceae) [26]

- Gynura nepalensis DC. (Asteraceae) [20, 22]

- Hypochaeris radicata L. (Asteraceae) [20, 22]

- Launaea nudicaulis (Linn.) Hook.f. (Asteraceae) [106]

- Rubus ellipticus Sm. (Rosaceae) [22]

- Rumex nepalensis Spreng. (Polygonaceae) [12]

- Schima wallichii (DC.) Korth. (Theaceae) [20, 22]

- Sonchus arvensis L. (Asteraceae) [20, 26, 22, 59, 66, 67, 105, 107] 
- Sonchus asper (L.) Hill (Asteraceae) [20, 22]

- Sonchus oleraceus L. (Asteraceae) [20, 26, 39, 41, 105]

- Sonchus sp. (Asteraceae) [20, 26, 39, 83,102, 107, 108, 109]

- Tridax procumbens L. (Asteraceae) [39, 61]

- Tridex sp. (Asteraceae) [110]

- Undet.: Asteraceae [71]

36. Hyperomyzus (Hyperomyzus) lactucae lactucae (Linnaeus, 1758)

= Nasonovia (Hyperomyzus) lactucae (Linnaeus, 1758) [78]

= Rhopalosiphum lactucae (Kaltenbach) [59]

- Cnicus argyracanthus (DC.) C.B. Clarke (Asteraceae) [71, 111]

- Sonchus arvensis L. (Asteraceae) [59]

- Sonchus asper (L.) Hill (Asteraceae) [59]

- Sonchus oleraceus L. (Asteraceae) [59, 78]

- Sonchus sp. (Asteraceae) $[44,71]$

- Taxus baccata L. (Taxaceae) [37, 71, 112]

- Undet.: Amaranthaceae [71]; Anacardiaceae [107]; Polygonaceae [70, 71)

37. Hyperomyzus sp.

- Rubus plicatus Weihe \& Nees (=Rubus fruticosus L.) (Rosaceae) [113]

38. Impatientinum (Impatientinum) asiaticum dalhousiensis Verma, 1969

= Macrosiphum (Sitobion) smilaceti dalhousiensis Verma, 1969 [75]

= Macrosiphum smilacicola Takahashi, 1924 [114]

= Impatientinum impatiens dalhousiensis Verma, 1969 [39, 69, 71, 115-118]

- Erigeron sp. (Asteraceae) [39, 71, 114, 117, 119]

- Fagopyrum acutatum (Lehm.) Mansf. ex K.Hammer (=Fagopyrum cymosum (Trev.) Meisn) (Polygonaceae) [39]

- Impatiens balsamina L. (Balsaminaceae) [39]

- Impatiens scabrida DC. (Balsaminaceae) $[12,71]$

- Impatiens sp. (= Balsam sp.) (Balsaminaceae) [39, 58, 71, 107, 119, 120]

- Rosa sp. (Rosaceae) [39, 71]

- Rumex sp. (Polygonaceae) [35, 61]

- Smilax parviflora Wall. (Smilacaceae) [69, 71, 121, 122]

- Smilax sp. (Smilacaceae) [35, 39, 61, 107]

39. Impatientinum (Impatientinum) asiaticum Nevsky, 1929

- Impatiens scabrida DC. (Balsaminaceae) [13]

- Impatiens sp. (Balsaminaceae) [13]

- Smilax sp. (Smilacaceae) [13]

40. Impatientinum (Impatientinum) balsamines (Kaltenbach, 1862)

- Impatiens sp. (Balsaminaceae) [13]

- Launaea sp. (Asteraceae) [13]

41. Impatientinum (Impatientinum) impatiens (Shinji, 1922)

- Impatiens bicornuta Wall. (Balsaminaceae) [41]

- Impatiens sp. (Balsaminaceae) [26, 69, 120]

- Smilax sp. (Smilacaceae) [37, 123]

- Undet.: Geraniaceae [124]

42. Impatientinum (Neoimpatientinum) smilaceti Agarwala, Mondal \& Raychaudhuri, 1982

- Salix macrophylla Andersson (Salicaceae) [125]

43. Indiaphis crassicornis Basu, 1969

- Rhododendron arboreum Sm. (Ericaceae) [35, 39]

- Rhododendron sp. (Ericaceae) [20, 22, 24, 35, 39]

44. Indiaphis indica (Ghosh, Verma \& Raychaudhuri, 1976)

= Neoacyrthosiphon indicum Ghosh, Verma \& Raychaudhuri, 1976 [17]

International J. Research Studies in Zoology (IJRSZ)

Page $\mid 46$ 
= Neoacyrthosiphon (Neoacyrthosiphon) indicum Ghosh, Verma \& Raychaudhuri, 1976

- Rhododendron sp. (Ericaceae) [23, 67, 71]

45.Indiaphis rostrata Ghosh \& Raychaudhuri, 1972

- Rhododendron sp. (Ericaceae) [20, 22, 24, 26]

46. Indiaphis setosa (Hille Ris Lambers \& Basu, 1966)

= Ericolophium (Neoacyrthosiphon) setosum Hille Ris Lambers \& Basu, 1966

= Neoacyrthosiphon setosum Hille Ris Lambers \& Basu, 1966 [17]

= Neoacyrthosiphon (Neoacyrthosiphon) setosum (Hille Ris Lambers \& Basu, 1966) [21]

- Pentapterygium serpens (Wight) Klotzsch (Ericaceae) [20, 21, 126]

47. Indoidiopterus geranii Chakrabarti, Ghosh \& Raychaudhuri, 1972

= Neotoxoptera geranii (Chowdhuri, Basu, Chakrabarti \& Raychaudhuri,1969) [127]

= Indoidiopterus geranii (Chowdhuri, Basu, Chakrabarti \& Raychaudhuri,1969) [17]

= Capitophorus geranii Chowdhuri, Basu, Chakrabarti \& Raychaudhuri,1969 [69, 71]

- Geranium divaricatum Ehrh. (Geraniaceae) [128]

- Geranium ocellatum Cambess. (Geraniaceae) [35]

- Geranium robertianum L. (Geraniaceae) [13]

- Geranium divaricatum Ehrh. (Geraniaceae) [69, 71, 120, 129]

- Geranium ocellatum Cambess. (Geraniaceae) [35, 39]

- Geranium wallichianum D.Don ex Sweet (Geraniaceae) [71]

- Geranium sp. (Geraniaceae) [35, 39, 41]

48. Indomasonaphis anaphalidis (Basu, 1964)

= Indomasonaphis indica Verma, 1971 [17]

= Indumasonaphis tuberculata Chakrabarti Mandal \& Raha, 1983

= Neomasonaphis anaphalidis (Basu, 1964) [127]

= Masonaphis (Neomasonaphis) anaphalidis Basu, 1964 [118]

= Masonaphis anaphalidis Basu, 1964 [35, 130]

= Neomasonaphis tuberculata (Chakrabarti Mandal \& Raha, 1983) [127 ]

- Ageratum conyzoides L. (Asteraceae) [20, 22, 37, 131]

- Anaphalis sp. (Asteraceae) [20, 26]

- Anaphalis triplinervis Benth. Ex Hance (Asteraceae) [132]

- Artemisia sp. (Asteraceae) [20, 22]

- Eurya japonica Thunb. (Ternstroemiaceae) [20, 22, 131]

- Gerbera sp. (Asteraceae) [40]

- Inula cappa (Buch.-Ham. Ex D. Don) DC. (Asteraceae) [22, 71, 119, 133]

- Morus alba L. (Moraceae) [20, 131]

- Oxalis corniculata L. (Oxalidaceae) [40]

- Rhododendron campestris ? (Ericaceae) [23]

- Rhododendron sp. (Ericaceae) [59, 98]

- Senecio rufinervis DC. (Asteraceae) [13]

- Senecio scandens Buch.-Ham. ex D. Don (Asteraceae) [20, 22, 131]

49. Indomasonaphis chakrabartii (Bhattacharya, 1991)

= Indumasonaphis chakrabartii Bhattacharya, 1991

= Neomasonaphis chakrabartii (Bhattacharya, 1991) [127]

- Inula sp. (Asteraceae) [134]

50. Indomasonaphis inulae (Ghosh \& Raychaudhuri, 1972)

= Indumasonaphis inulae (Ghosh \& Raychaudhuri, 1972) [23]

= Masonaphis (Neomasonaphis) inulae Ghosh \& Raychaudhuri, 1972 [71, 135]

= Neomasonaphis inulae (Ghosh \& Raychaudhuri, 1972) [127]

- Inula cappa (Buch.-Ham. Ex D. Don) DC. (Asteraceae) [20, 22, 107, 135]

- Inula cuspidata Clarke (Asteraceae) [13]

- Rhododendron sp. (Ericaceae) [13, 20, 22, 135]

- Rosa sp. (Rosaceae) [71] 
51. Indomasonaphis rumicis (Chakrabarti \& Raychaudhuri, 1975)

= Masonaphis (Neomasonaphis) rumicis Chakrabarti \& Raychaudhuri, 1975

= Neomasonaphis rumicis (Chakrabarti \& Raychaudhuri, 1975) [127]

- Gerbera sp. (Asteraceae) [41, 42]

- Oxalis sp. (Oxalidaceae) [42]

- Oxyria digyna (L.) Hill (Polygonaceae) [37, 41]

- Rumex sp. (Polygonaceae) [37, 41, 42]

52. Indomasonaphis vasesiphon (Das \& Ghosh, 2002)

= Neomasonaphis vasesiphon Das \& Ghosh, 2002

- Scenocio sp. (Asteraceae) [136]

53. Indomegoura indica (van der Goot, 1916)

= Rhopalosiphum indicum van der Goot, 1916 [59]

- Unidentified plants [59, 98]

54. Indomyzus glaricae Sathe \& Jadhav, 2008§

- Gliricidia maculata (Kunth) Kunth ex Walp. (Fabaceae) [137]

55. Indomyzus sensoriatus Ghosh, Ghosh \& Raychaudhuri, 1971

- Unidentified plants [20, 22, 94]

56. Ipuka dispersa (van der Goot, 1917)

= Ipuka dispersum (van der Goot, 1917) [17]

= Aulacorthum (Neomyzus) dispersum van der Goot, 1917 [59, 138]

- Emilia sonchifolia L. (Asteraceae) [59, 62, 138]

57. Jacksonia campanulata Chakrabarti \& Raychaudhuri, 1978

- Campanula dimorphantha Schweinf. (=Campanula benthamii Wall. ex Kitam.) (Campanulaceae) [139]

- Campanula pallida var. pallida Wall. (=Campanula colorata Wall.) (Campanulaceae) [39, 140]

58. Jacksonia papillata Theobald, 1923

- Poa sp. (Poaceae) [71, 85]

59. Jacksonia sikkimensis Ghosh, Basu \& Raychaudhuri, 1977

- Undet.: Poaceae [20, 26, 141]

60. Jacksonia sp.

- Sarcococca coriacea (Hook.) Sweet (=Sarcococca pruniformis Lindl.) (Buxaceae) [37, 39, 142]

61. Kaochiaoja arthraxonis (Takahashi, 1921)

= Micromyzus granotiae Ghosh, Ghosh \& Raychaudhuri, 1970 [17]

= Aulacorthum (Neomyzus) arthraxonis Takahashi, 1921 [64]

- Granotia sp. (Asteraceae) [20, 22]

- Unidentified plant [64]

62. Liosomaphis atra Hille Ris Lambers, 1966

(= Wahlgreniella neoempetri Ghosh, Basu \& Raychaudhuri, 1971)

- Berberis aristata DC. (Berberidaceae) [39, 71]

- Berberis asiatica Roxb. ex DC. (Berberidaceae) [143]

- Berberis lycium Royle (= Berberis pseudoumbellata Parker) (Berberidaceae) [58, 144]

- Berberis sp. (Berberidaceae) [39, 61, 69, 70, 71, 107]

- Launaea pinnatifida Cass. (=Launaea nepalense auct. nonn.) (Asteraceae) [13]

- Polygonum sp. (Polygonaceae) [71]

63. Liosomaphis berberidis (Kaltenbach, 1843)

= Liosomaphis berberdis Verma \& Das, 1992 [75]

- Berberis lycium Royle (Berberidaceae) [145]

- Berberis umbellata Wall. ex G. Don. (Berberidaceae) [20]

International J. Research Studies in Zoology (IJRSZ)

Page $\mid 48$ 


\section{Liosomaphis himalayensis Basu, 1964}

- Berberis aristata DC. (Berberidaceae) [39]

- Berberis asiatica Roxb. ex DC. (Berberidaceae) [20, 35, 39, 40]

- Berberis lycium Royle (Berberidaceae) $[69,146]$

- Berberis sp. (Berberidaceae) [63]

- Berberis umbellata Wall. ex G. Don. (Berberidaceae) [20, 22, 38, 132]

- Berberis wallichiana DC. (Berberidaceae) [20]

- Brassica napus L. (Brassicaceae) [26]

- Brassica sp. (Brassicaceae) [20, 22, 26, 39-41, 69, 71, 93,107]

- Fagopyrum sp. (Polygonaceae) [147]

- Launaea pinnatifida Cass. (=Launaea nepalense auct. nonn.) (Asteraceae) [13]

- Launaea sp. (Asteraceae) [13]

- Prunus spinosa L. (=Crataegus spinosa auct.) (Rosaceae) [26]

- Raphanus sativus L. (Brassicaceae) [26]

- Rumex sp. (Polygonaceae) [63]

- Viburnum mullaha Buch.-Ham. ex D. Don (=Viburnum stellulatum Wall. ex DC.) (Adoxaceae (=Caprifoliaceae)) [12]

- Viburnum sp. (Adoxaceae) [12]

65. Liosomaphis ornata Miyazaki, 1971

- Berberis lycium Royle (Berberidaceae) [143 ]

66. Liosomaphis sp.

- Berberis zebiliana Slmeidc. (Berberidaceae) [144]

- Prinsepia sp. (Rosaceae) [71, 111]

- Tecoma sp. (Bignoniaceae) [89]

67. Lipaphis (Lipaphis) erysimi (Kaltenbach, 1843)

= Siphocoryne indobrassicae Das, 1918

- Acacia catechu (L.f.) Willd. (Fabaceae) [148]

- Allium sativum L. (Alliaceae) [89]

- Beta vulgaris L. (Chenopodiaceae) [149]

- Brassica carinata A. Braun (Brassicaceae) [150]

- Brassica juncea L. Czern. (Brassicaceae) [20, 22, 107, 151]

- Brassica juncea var. juncea L. Czern. (= Brassica juncea var. raya L.) (Brassicaceae) [152]

- Brassica juncea var. rugosa (Roxb.) N. Tsen \& S.N. Lee (L.) Czern. (Brassicaceae) [153]

- Brassica napus L. (Brassicaceae) [20, 22, 26, 30, 98, 83, 103]

- Brassica nigra (L.) W.D.J. Koch (Brassicaceae) [67, 83, 98]

- Brassica oleracea L. (Brassicaceae) [20, 22, 30, 37, 39, 60, 98],

- Brassica oleracea L. var. viridis (=Brassica oleracea var. acephala) DC.) (Brassicaceae) [84]

- Brassica oleracea var. botrytis L. (=Brassica oleracea var. cauliflora (misnomen.) (Brassicaceae) [83, 67, 97, 103]

- Brassica oleracea var. capitata L. (Brassicaceae) [27, 67, 84, 97]

- Brassica oleracea var. gongyloides L. (Brassicaceae) [27, 67, 151, 154]

- Brassica rapa L. (Brassicaceae) [59, 84, 97]

- Brassica rapa subsp. campestris (L.) A.R. Clapham (= Brassica campestris L.) (Brassicaceae) [28, 37, 39, 67, 84, 103, 149]

- Brassica sp. (Brassicaceae) [20, 22, 26, 28, 39, 69, 81, 82, 98, 149, 151]

- Calendula sp. (Asteraceae) [20, 22, 97, 103]

- Calotropis gigantea (L.) W.T. Aiton (Apocynaceae (=Asclepiadaceae)) [155]

- Calotropis procera (Aiton) W.T. Aiton (Apocynaceae) [89]

- Cardamine hirsuta L. (Brassicaceae) [67]

- Cleome gyandra L. (=Cleome pentaphylla L.; Gynandropsis pentaphylla (L.) DC) (Cleomaceae) $[62,66]$

- Daucus carota L. (Apiaceae) [156]

- Drymaria cordata (L.) Willd. ex Schult. (Caryophyliaceae) [20] 
- Emilia sonchifolia L. (Asteraceae) [20]

- Eruca sativa Miller (Brassicaceae) [59]

- Erysimum cheiri (L.) Crantz (=Cheiranthus cheiri L.) (Brassicaceae) [59]

- Ficus heterophylla L.f. (Moraceae) [22, 97, 103]

- Foeniculum vulgare Mill. (Apiaceae) [83]

- Iberis amara L. (Brassicaceae) [20, 22, 39, 71, 111]

- Lactuca sativa L. (Asteraceae) [20, 22, 97, 103]

- Lepidium didynum L. (=Coronopus dydimus (L.) Sm.) (Brassicaceae) [59]

- Lycopersicon esculentum Mill. (Solanaceae) [79, 89, 98, 149]

- Matthiola incana (L.) W. T. Aiton (=Guettarda incana auct. nonn.) (Brassicaceae) [39, 84]

- Momordica charantia L. (Cucurbitaceae) [156]

- Nasturtium officinale R. Br. (Brassicaceae) [13]

- Nicotiana tabacum L. (Solanaceae) [22, 79, 83, 149]

- Petunia alba Hort. Ex Ferg. \& Ottl. (Solanaceae) [103, 157]

- Pyrus communis L. (Rosaceae) [22]

- Raphanus sativus L. (Brassicaceae) [26, 97, 103, 149, 154, 157]

- Rhododendron sp. (Ericaceae) [22]

- Rorippa indica (L.) Hiern (=Nasturtium indicum (L.) DC.) (Brassicaceae) [20, 22]

- Salix sp. (Salicaceae) [83]

- Solanum melongena L. (Solanaceae) [30]

- Solanum tuberosum L. (Solanaceae) [69, 79, 82]

- Spinacia oleracea L. (Chenopodiaceae) [103]

- Terminalia paniculata Roth (Combretaceae) [148]

- Trichosanthes cucumerina var. anguina (L.) (=Trichosanthes anguina L.) (Cucurbitaceae) [156]

- Trifolium alexandrinum L. (=Triticum alexandrum auct. nonn.) (Fabaceae) [89]

- Triticum aestivum L. (Poaceae) [89]

- Tropaeolum majus L. (Tropaeolaceae) [20, 22, 97, 98, 103]

- Withania somnifera (L.) Dunal (Solanaceae) [30, 98]

- Undet.: Araliaceae [59, 158]; Brassicaceae [37, 44, 66, 69, 74, 79, 103, 158]; Fabaceae [69]

68. Lipaphis (Lipaphis) pseudobrassicae (Davis, 1914)

= Siphocoryne pseudobrassicae (Davis, 1914) [59, 919]

= Rhopalosiphum pseudobrassicae (Davis, 1843) [60, 74, 160]

- Beta vulgaris L. (Chenopodiaceae) [59, 149]

- Brassica juncea L. Czern. (Brassicaceae) [59, 161]

- Brassica nigra (L.) W.D.J. Koch (Brassicaceae) [59, 160]

- Brassica oleracea L. (Brassicaceae) [60]

- Brassica oleracea var. botrytis L. (=Brassica oleracea var. cauliflora (misnomen.) (Brassicaceae) [59]

- Brassica oleracea var. capitata L. (Brassicaceae) [59, 60]

- Brassica oleracea var. gongyloides L. (Brassicaceae) [59]

- Brassica rapa L. (Brassicaceae) [59]

- Brassica rapa subsp. campestris (L.) A.R. Clapham (= Brassica campestris L.) (Brassicaceae) $[59,149]$

- Calotropis gigantea (L.) W.T. Aiton (Apocynaceae) [59]

- Cleome gyandra L. (=Cleome pentaphylla L.; Gynandropsis pentaphylla (L.) DC) (Cleomaceae) [59]

- Eruca sativa Miller (Brassicaceae) [59]

- Erysimum cheiri (L.) Crantz (=Cheiranthus cheiri L.) (Brassicaceae) [59]

- Lepidium didynum L. (=Coronopus dydimus (L.) Sm.) (Brassicaceae) [59]

- Lepidium sativum L. (Brassicaceae) [59]

- Lycopersicon esculentum Mill. (Solanaceae) [59,149]

- Matthiola incana (L.) W. T. Aiton (=Guettarda incana auct. nonn.) (Brassicaceae) [162]

- Nasturtium sp. (Brassicaceae) [59]

- Nicotiana tabacum L. (Solanaceae) [59, 149] 
- Raphanus sativus L. (Brassicaceae) [59, 60, 149, 162]

- Sesamum indicum L. (Pedaliaceae) [59]

- Sisymbrium irio L. (Brassicaceae) [59]

- Solanum tuberosum L. (Solanaceae) [59]

- Undet.: Araliaceae [59]; Brassicaceae [74]

69. Longicaudus cornutus Chakrabarti \& Banerjee, 1991

- Thalictrum sp. (Ranunculaceae) [163]

70. Longicaudus dunlopi Hille Ris Lambers, 1965

- Thalictrum chelidonii DC. (Ranunculaceae) [36, 39, 40, 41]

71. Longicaudus jakhuensis (Raychaudhuri, Ghosh \& Das, 1980)

= Senisetotarsaphis jakhuensis Raychaudhuri, Ghosh \& Das, 1980 [17]

= Longicaudus himalayensis Hille Ris Lambers, 1965 [13]

- Quercus sp. (Fagaceae) [70, 215]

- Rosa sp. (Rosaceae) [188]

- Rubus sp. (Rosaceae ) [17]

- Tephrosia sp. (Fabaceae) [13, 139]

72. Longicaudus kumauni Chakrabarti \& Banerjee, 1991

- Thalictrum sp. (Ranunculaceae) [163]

73. Longisiphoniella subterranea Chakrabarti, Saha \& Mandal, 1984 (1988)

- Artemisia sp. (Asteraceae) [139]

- Conyza bonariensis (L.) Cronq. (=Erigeron bonariensis L.) (Asteraceae) [164]

- Cynoglossum wallichii G. Don var. glochidiatum (Wall. ex Benth.) Kazmi (=Cynoglossum glochidiatum Wall. ex Benth.) (Boraginaceae) [139]

- Poa sp. (Poaceae) [139]

- Rumex sp. (Polygonaceae) [139]

74. Macromyzella polypodicola (Takahashi, 1921)

= Macromyzus polypodicoala $($ Takahashi, 1921) [165 ]

= Macromyzus polypodicola $($ Takahashi, 1921) $[18,65,80]$

- Asplenium esculentum (Retz.) (Aspleniaceae) [20, 24, 165, 166, 167]

- Asplenium sp. (Aspleniaceae) [20]

- Cheilanthes farinosa Kaulf. (Pteridaceae) [168]

- Salix sp. (Salicaceae) [65]

75. Macromyzus (Anthracosiphoniella) maculatus (Basu, 1969)

= Macr omyzus (Anthracosiphoniella) maculatum (Basu, 1969)

= Anthracosiphoniella maculata Basu, 1969 [18, 29, 170]

- Asplenium esculentum (Retz.) (Aspleniaceae) [20, 24, 166]

- Athyrium sp. (Dryopteridaceae (=Athyriaceae)) [20, 24, 166, 170]

- Dryopteris mollis (Jacq.) Hieron. (Dryopteridaceae) [20]

- Eriosorus aurita ? (Pteridaceae) [26]

76. Macromyzus (Macromyzus) indicus David \& Narayanan, 1968

- Unidentified plants [71, 171]

77. Macromyzus (Macromyzus) manoji Raha \& Raychaudhuri, 1978

= Macromyzus woodwardiae (Takahashi, 1921) [80, 172]

- Asplenium esculentum (Retz.) (Aspleniaceae) [165]

- Cheiranthus varius Sibth. \& Sm. (Brassicaceae) [173]

- Indet.: Fern [172]

78. Macromyzus (Macromyzus) woodwardiae (Takahashi, 1921)

- Asplenium adiantum-nigrum L. (Aspleniaceae) [20]

- Asplenium adientum-nigrum L. (Aspleniaceae) [167]

- Asplenium auriculatum Sw. (Aspleniaceae) [167] 
- Asplenium curicularium ? (Aspleniaceae) [20, 24, 166]

- Asplenium esculentum (Retz.) (Aspleniaceae) [20, 24, 165, 166]

- Athyrium anisopterum Christ (=Athyrium macrocarpum auct. nonn. (Blume) Beddome) (Dryopteridaceae (=Athyriaceae)) [20]

- Athyrium sp. (Dryopteridaceae (=Athyriaceae)) [20]

- Cheiranthus varius Sibth. \& Sm. (Brassicaceae) [20]

- Dicheria alata ? (Polypodiaceae) [20]

- Dryopteris mollis (Jacq.) Hieron. (=Nephrodium mollis auct. non.) (Dryopteridaceae) [20,167]

- Poa sp. (Poaceae) [13]

- Polypodium sp. (Polypodiaceae) [20]

79. Macrosiphoniella (Chosoniella) spinipes Basu, 1968

= Macrosiphum (Macrosiphum) spinipes (Basu, 1968) [172]

= Macrosiphoniella spinipes rhododendri Ghosh, Basu \& Raychaudhuri, 1969

- Artemisia caruifolia Buch.-Ham. Ex Roxb. (Asteraceae) [26]

- Artemisia sp. (Asteraceae) [20, 26, 30, 34, 98]

- Artemisia vulgaris L. (Asteraceae) [20, 26, 68, 83, 172, 174]

- Chrysanthemum sp. (Asteraceae) [19]

- Rhododendron arboreum Sm. (Ericaceae) [20, 174, 175]

80. Macrosiphoniella (Macrosiphoniella) alatavica (Nevsky, 1928)

= Macrosiphoniella alatavica (Nevsky, 1928) [17]

= Macrosiphoniella lambersi Verma, 1971 [78, 176]

- Artemisia scoparia Waldst. \& Kit (Asteraceae) [78, 121]

- Artemisia sp. (Asteraceae [72]

80a. Macrosiphoniella (Macrosiphoniella) artemisiae artemisiae (Boyer de Fonscolombe, 1841)

81. Macrosiphoniella (Macrosiphoniella) formosartemisiae Takahashi, 1921

= Macrosiphoniella formosartemisiae Takahashi, 1921[17]

- Artemisia vulgaris L. (Asteraceae) [20, 22, 108, 174]

- Chrysanthemum sp. (Asteraceae) [13]

- Conyza japonica (Thunb.) DC. (Asteraceae) [22]

- Eurya japonica Thunb. (Ternstroemiaceae) [20, 174]

82. Macrosiphoniella (Macrosiphoniella) himalayana Saha \& Chakrabarti, 1988

- Artemisia sp. (Asteraceae) [177 ]

83. Macrosiphoniella (Macrosiphoniella) kalimpongensis Basu \& Raychaudhuri, 1976

= Macrosiphoniella kalimpongense Basu \& Raychaudhuri, 1976 [107]

- Artemisia sp. (Asteraceae) [174]

- Artemisia vulgaris L. (Asteraceae) [20, 107, 174]

84. Macrosiphoniella (Macrosiphoniella) kikungshana Takahashi, 1937

= Macrosiphoniella matsumurana Ghosh, Basu \& Raychaudhuri, 1970 [17]

= Macrosiphoniella (Sinosiphoniella) hikosanensis Moritsu, $1949 *$ : Ghosh A.K., 1973

- Artemisia caruifolia Buch.-Ham. Ex Roxb. (Asteraceae) [26]

- Artemisia caruifolia Buch.-Ham. Ex Roxb. (Asteraceae) [26]

- Artemisia sp. (Asteraceae) [26, 63]

- Artemisia vulgaris L. (Asteraceae) [20, 22, 64, 80, 91, 147, 174]

- Asclepias curassavica L. (Apocynaceae) [20, 26, 174]

- Chrysanthemum sp. (Asteraceae) [147]

- Eupatorium odoratum L. (Asteraceae) [22]

- Indet.: Asteraceae [71]

85. Macrosiphoniella (Macrosiphoniella) pseudoartemisiae Shinji, 1933

= Macrosiphoniella pseudoartemisiae Shinji, 1933[17]

- Artemisia absinthium Linn. (Asteraceae) [144, 178]

- Artemisia scoparia Waldst. \& Kit (Asteraceae) [179] 
- Artemisia sp. (Asteraceae) [20, 22, 26, 37, 39, 98, 105, 147, 174, 180]

- Artemisia vulgaris L. (Asteraceae) [25, 26, 39, 69, 93, 142, 146, 174]

- Chrysanthemum sp. (Asteraceae) [13]

- Glebionis coronaria (L.) Cass. ex Spach (=Chrysanthemum coronarium L.) (Asteraceae) [181]

- Ixora coccinea L. (Rubiaceae) [106]

86. Macrosiphoniella (Macrosiphoniella) sanborni (Gillette, 1908)

= Macrosiphum sanborni (Gillette, 1908) [74, 182]

= Macrosiphoniella eastopi Kulkarni, 1980 (1981) [127 ]

- Artemisia sp. (Asteraceae) [20, 26, 174]

- Artemisia vulgaris L. (Asteraceae) [184]

- Calotropis gigantea (L.) W.T. Aiton (Apocynaceae) [103, 155]

- Chrysanthemum indicum L. (Asteraceae) [20, 39, 59, 66, 81, 174]

- Chrysanthemum xmorifolium Ramat. (=Chrysanthemum sinense Sabine ex Sweet) (Asteraceae) $[59,69,144,146]$

- Chrysanthemum sp. (Asteraceae) [34, 67, 69, 71, 74, 79, 83, 107, 147, 149, 151, 174]

- Dendranthema grandiflora (Asteraceae) [176a]

- Delonix regia (Bojer ex Hook) Raf. (Fabaceae) [89, 90]

- Geranium sp. (Geraniaceae) [22]

- Glebionis coronaria (L.) Cass. ex Spach (=Chrysanthemum coronarium L.) (Asteraceae) [20, $22,26,82,174]$

- Montanoa bipinnatifida (Kunth) K. Koch (Asteraceae) [22]

- Parthenium hysterophorus L. (Asteraceae) [106]

- Vernonia sp. (Asteraceae) [59, 74]

- Undet.: Asteraceae) [71, 184]

87. Macrosiphoniella (Macrosiphoniella) sikkimartemisiae Agarwala \& Raychaudhuri, 1977

$=($ Macrosiphoniella $)$ sikkimartemisiae Agarwala \& Raychaudhuri, 1977 [17]

- Artemisia sp. (Asteraceae) [26, 185]

88. Macrosiphoniella (Macrosiphoniella) sudhakaris Banerjee, Ghosh \& Raychaudhuri, 1969

= Macrosiphoniella) sudhakaris Banerjee, Ghosh \& Raychaudhuri, 1969 [17]

- Achillea millefolium L. (Asteraceae) [39, 142]

89. Macrosiphoniella (Macrosiphoniella) yomogifoliae (Shinji, 1924)

= Macrosiphoniella) yomogifoliae (Shinji, 1924) [17]

= Macrosiphoniella plutea Buckton [186]

= Macrosiphoniella artemisiae yomogifoliae Shinji [69, 71, 120]

- Artemisia caruifolia Buch.-Ham. Ex Roxb. (Asteraceae) [26]

- Artemisia sp. (Asteraceae) [20, 22, 26, 83, 107, 147, 174, 183, 186]

- Artemisia vestita Wall (Asteraceae) [39]

- Artemisia vulgaris L. (Asteraceae) [20, 22, 26, 39, 59, 65, 66, 71, 83, 174]

- Bidens bipinnata L. (= Bidens wallichii DC.) (Asteraceae) [39, 69, 71, 120, 129]

- Gnaphalium sp. (Asteraceae) [13]

90. Macrosiphoniella (Phalangomyzus) grandicauda Takahashi \& Moritsu, 1963

= Macrosiphoniella grandicauda Takahashi \& Moritsu, 1963 [17]

- Ainsliaea aptera DC. (Asteraceae) [13]

- Artemisia sp. (Asteraceae) [20, 174]

- Eurya japonica Thunb. (Ternstroemiaceae) [109, 131, 174]

- Eurya sp. (Ternstroemiaceae) [20]

91. Macrosiphoniella (Phalangomyzus) oblonga (Mordvilko, 1901)

= Macrosiphoniella oblonga (Mordvilko, 1901) [94, 109]

- Artemisia sp. (Asteraceae) [26]

- Eurya japonica Thunb. (Ternstroemiaceae) [36, 109, 131] 
92. Macrosiphoniella (Phalangomyzus) paraoblonga Basu \& Raychaudhuri, 1976b

- Artemisia sp. (Asteraceae) [174]

93. Macrosiphoniella (Sinosiphoniella) hikosanensis Moritsu, 1949

= Macrosiphoniella hikosanensis Moritsu, 1949 [165]

= Macrosiphoniella hikkosanensis Moritsu, 1949 [17]

- Artemisia sp. (Asteraceae) [13]

- Artemisia vulgaris L. (Asteraceae) [71, 187]

94. Macrosiphoniella sp.

- Achillea millefolium L. (Asteraceae) [144]

- Artemisia sp. (Asteraceae) [63]

- Artemisia vulgaris L. (Asteraceae) [67]

- Chrysanthemum sp. (Asteraceae) [187]

95. Macrosiphum (Macrosiphum) centranthi Theobald, 1915

= Macrosiphum euphorbiae (Thomas, 1878)* [101, 188, 189]

- Chrysanthemum xmorifolium Ramat. (=Chrysanthemum sinense Sabine ex Sweet) (Asteraceae) [117]

- Chrysanthemum sp. (Asteraceae) [66, 190]

- Cineraria sp. (Asteraceae) [117]

- Echeveria sp. (Crassulaceae) [87, 117]

- Mangifera indica L. (Anacardiaceae) [66, 190]

- Pisum sativum L. (Fabaceae) [117]

- Ranunculus sp. (Ranunculaceae) [117]

- Rosa sp. (Rosaceae) [66, 190]

- Silene coel-rosa (L.) Godr. (= Agrostemma coelirosa L.) (Caryophyliaceae) [117, 191]

- Solanum tuberosum L. (Solanaceae) [101]

- Tibouchina semidecandra (Schrank \& Mart. Ex DC.) Cogn. (Melastomaceae) [117]

- Tibouchina sp. (Melastomaceae) [117]

96. Macrosiphum (Macrosiphum) euphorbiae (Thomas, 1878)

- Alcea rosea L. (Malvaceae) [69, 71]

- Chrysanthemum sp. (Asteraceae) [71]

- Mangifera indica L. (Anacardiaceae) [71]

- Rosa sp. (Rosaceae) [176a]

- Solanum tuberosum L. (Solanaceae) $[69,71,101]$

- Verbena sp. (Verbenaceae) [69, 71]

97. Macrosiphum (Macrosiphum) fagopyri Ghosh \& Raychaudhuri, 1972

= Macrosiphum fagopyri Ghosh \& Raychaudhuri, 1972

= Macrosiphum (Sitobion) fagopyri Ghosh \& Raychaudhuri, 1972 [17, 118]

- Fagopyrum acutatum (Lehm.) Mansf. ex K.Hammer (=Fagopyrum cymosum (Trev.) Meisn) (Polygonaceae) [20]

- Fagopyrum sp. (Polygonaceae) [13, 20, 117, 135]

98. Macrosiphum hellebori Theobald \& Walton, 1923

- Chrysanthemum sp. (Asteraceae) [59]

- Echeveria sp. (Crassulaceae) [59]

- Mangifera indica L. (Anacardiaceae) [59]

- Ranunculus sp. (Ranunculaceae) [59, 138]

- Rosa sp. (Rosaceae) [59]

- Silene coel-rosa (L.) Godr. (= Agrostemma coelirosa L.) (Caryophyliaceae) [59]

- Solanum tuberosum L. (Solanaceae) [101]

99. Macrosiphum (Macrosiphum) pachysiphon Hille Ris Lambers, 1966

- Berberis sp. (Berberidaceae) [192]

- Chrysanthemum sp. (Asteraceae) [22] 
- Potentilla atrosanguinea var. argyrophylla (Wall. ex Lelum.) Griersen \& Long (=Potentilla argyrophylla Wall. ex Lelum.) (Rosaceae) [20, 22, 39, 69, 71, 120, 129]

- Rosa sp. (Rosaceae) [39, 61, 69, 71, 117, 129, 190]

- Rubus ellipticus Sm. (Rosaceae) [20, 22]

- Rubus macilentus Cambess (Rosaceae) [20, 22]

- Rubus nivens Rhunb. (=Rubus lasiocarpus Sm.) (Rosaceae) [20, 22, 39, 71, 117]

- Rubus rosifolius Sm. (=Rubus rosaefolia auct.) (Rosaceae) [19, 20, 22, 67]

- Rubus sp. (Rosaceae) [107, 117, 124, 190]

- Rumex hastatulus Baldwin (Polygonaceae) [39, 69, 71, 129]

- Triticum aestivum L. (Poaceae) [83]

- Triticum aestivum ssp. aestivum L. (=Triticum vulgare Vill.) (Poaceae) [22]

- Undet.: Rosaceae) [26, 105, 117]

100. Macrosiphum (Macrosiphum) pseudogeranii Chakrabarti \& Raychaudhuri, 1974

= Macrosiphum (Neomacrosiphum) pseudogeranii Chakrabarti \& Raychaudhuri, 1974 [17]

= Macrosiphum geranii (Oestlund, 1887)* [71, 114, 115, 129]

- Geranium lucidum L. (Geraniaceae) [71, 120, 129]

- Geranium nepalense Sweet (Geraniaceae) [117, 147]

- Geranium sp. (Geraniaceae) [39, 114]

- Geranium wallichianum D.Don ex Sweet (Geraniaceae) [71]

- Polygonum sp. (Polygonaceae) [107]

101. Macrosiphum (Macrosiphum) rosae rosae (Linnaeus, 1758)

= Macrosiphum (Macrosiphum) rosae (Linnaeus, 1758) [17]

= Siphonophora rosae (Linnaeus, 1758) [193]

- Bignonia sp. (Bignoniaceae) [180]

- Cheilanthes sp. (Pteridaceae) [180]

- Fagopyrum sp. (Polygonaceae) [22]

- Inula cappa (Buch.-Ham. Ex D. Don) DC. (Asteraceae) [13]

- Pyrus communis L. (Rosaceae) [117]

- Rosa americana Breit. (Rosaceae) [20, 22,]

- Rosa canina L. (Rosaceae) [20, 22, 26, 181]

- Rosa indica L. (Rosaceae) [194]

- Rosa macrophylla Lindl. (Rosaceae) [20, 22, 41]

- Rosa sp. (Rosaceae) [26-28, 39, 66, 69, 81, 98, 105, 117, 190]

102. Macrosiphum (Macrosiphum) koyenensis Sathe \& Jadhav, 2008§

= Macrosiphum koyenensis Sathe \& Jadhav, 2008

- Rosa multiflora Thunb.(Rosaceae) [137]

\section{Macrosiphum sp.}

- Argyroxiphium sp. (Asteraceae) [71, 111]

- Chrysanthemum sp. (Asteraceae) [195]

- Lactuca sativa L. (Asteraceae) [97, 103]

- Lathyrus odoratus L. (Fabaceae) [59]

- Linum usitatissimum L. (Linaceae) [103]

- Poa sp. (Poaceae) [71, 111]

- Rosa sp. (Rosaceae) [67]

- Solanum tuberosum L. (Solanaceae) [187]

- Undet.: Fabaceae [59]; Poaceae [82]

104. Matsumuraja capitophoroides Hille Ris Lambers, 1966

- Clematis buchananiana DC. (Ranunculaceae) [13]

- Euphorbia hirta L. (Euphorbiaceae) [196]

- Poa annua L. (Poaceae) [197]

- Polygonum flaccidum Roxb. (=Polygonum serrulatum Lagasca) (Polygonaceae) [20, 22]

- Rosa sp. (Rosaceae) [39, 61, 71, 109, 190] 
- Rubus ellipticus Sm. (Rosaceae) [20, 22,180]

- Rubus macilentus Cambess (Rosaceae) [39, 69, 71, 120, 129]

- Rubus nivens Rhunb. (=Rubus lasiocarpus Sm.) (Rosaceae) [39, 67]

- Rubus niveus Thunb. (Rosaceae) [39, 41]

- Rubus rosifolius Sm. (=Rubus rosaefolia auct.) (Rosaceae) [20, 22, 180]

- Rubus rugosus Sm. (Rosaceae) [180]

- Rubus xylenica ? (Rosaceae) [20, 22]

- Rubus sp. (Rosaceae) [34, 83, 190]

- Indet.: Poaceae [129]

105. Matsumuraja intermedia Saha, Samanta, Pramanik \& Raychaudhuri, 1983

- Indet.: Urticaceae [198]

106. Matsumuraja nuditerga Hille Ris Lambers, 1965

- Pilea sp. (Urticaceae) [20, 22]

- Undet.: Urticaceae [20, 22]

107. Matsumuraja rubifoliae Takahashi, 1931

- Rubus sp. (Rosaceae) [199]

108. Matsumuraja urticae Ghosh, Ghosh \& Raychaudhuri, 1971

= Matsumuraja indica Ghosh, Ghosh \& Raychaudhuri, 1971

- Rubus sp. (Rosaceae) [123]

- Undet.: Urticaceae [20, 22, 123]

109. Matsumuraja spp.

- Boehmeria clidemioides var. clidemioides Miq. (= Boehmeria sidifolia Wedd.) (Urticaceae) [67]

- Rubus nivens Rhunb. (=Rubus lasiocarpus Sm.) (Rosaceae) [67]

110. Megoura dooarsis (Ghosh \& Raychaudhuri, 1969)

= Neomegouropsis dooarsis (Ghosh \& Raychaudhuri, 1969) [17]

= Megoura pallipes Basu A.N., 1969 [18, 22, 161]

= Megouroparsus dooarsis Ghosh \& Raychaudhuri, 1969 [35, 39, 69, 71, 200]

- Asplenium trichomanes L. (Aspleniaceae) [71]

- Indigofera dosua Buch.-Ham. ex D. Don (Fabaceae) [20]

- Indigofera heterantha Wall. ex Brandis (Fabaceae) [58]

- Indigofera sp. (Fabaceae) [18, 22]

- Indigofera zollingeriana Miq. (=Indigofera teysmannii Miq.) (Fabaceae) [20, 22, 64]

- Pyrus communis L. (Rosaceae) [161]

- Tephrosia purpurea (L.) Pers. (Fabaceae) [39, 61, 110]

- Undet.: Fabaceae [20, 69, 71, 200]

111. Megoura lespedezae (Essig \& Kuwana, 1918)

= Megoura abnormis Ghosh, L.K. 1970 [127]

= Neomegouropsis cajanae Ghosh, Ghosh \& Raychaudhuri (1970) 1971 [127]

- Cajanus cajan (L.) Millsp. (Fabaceae) [20, 22, 30, 36, 37, 83, 98, 201]

- Desmodium sp. (Fabaceae) [124, 201]

- Desmodium triflolium (L.) DC. (Fabaceae) [20, 22, 167]

- Undet.: Fabaceae $[44,71]$

112. Megoura sp.

- Indigofera dregeana F. Mey (=Indigofera gerardiana Wall. ex Baker) (Fabaceae) [69-71]

113. Metopolophium (Metopolophinum) darjeelingense Ghosh, 1970

= Metopolophium (Metopolophinum) darjeelingensis Ghosh, 1970 [17]

= Metopolophium (Neometopolophium) davidi Raychaudhuri, Ghosh \& Basu, 1975 (1978)

- Hypericum sp. (Hypericaceae) [20, 202]

- Undet.: Acanthaceae [20] 
114. Metopolophium (Metopolophium) chandrani (David \& Narayanan, 1968)

= Acyrthosiphon (Metopolophium) chandrani David \& Narayanan, 1968 [71, 203]

= Acyrthosiphon (Metopolophium) simlaense Chakrabarti \& Raychaudhuri, 1974 [71, 116, 119]

= Metopolophium (Metopolophium) graminum Raychaudhuri, Ghosh \& Das, 1980 [17]

= Metopolophium (Metopolophium) simlaense (Chakrabarti \& Raychaudhuri, 1974) [17]

- Bromus catharticus var. catharticus Vahl. (=Bromus uniloides Kunth) (Poaceae) [171]

- Launaea sp. (Asteraceae) [204, 205]

- Poa annua L. (Poaceae) [71, 171]

- Rosa sp. (Rosaceae) [71]

- Rubus ellipticus Sm. (Rosaceae) [206]

- Rubus opulifolius Bertol. (Rosaceae) [171]

- Undet.: Poaceae [71, 107]; unidentified [71, 116, 119]

115. Metopolophium (Metopolophium) darjilingense Raychaudhuri, Ghosh \& Basu, 1975

= Metopolophium (Microlophium) darjilingense Raychaudhuri, Ghosh \& Basu, 1975 [17]

- Undet.: Acanthaceae [20]

116. Metopolophium (Metopolophium) dirhodum (Walker, 1849)

= Acyrthosiphon (Metopolophium) dirhodum (Walker, 1849) [35, 39, 71, 200]

- Anthoxanthum hookeri (Griseb.) Rendle (=Hierochloe hookeri (Grisch) Clarke) (Poaceae) [35]

- Bromus catharticus var. catharticus Vahl. (=Bromus uniloides Kunth) (Poaceae) [35, 39]

- Bromus sp. (Poaceae) [13]

- Dactylus sp. (Poaceae) [71]

- Triticum aestivum ssp. aestivum L. (=Triticum vulgare Vill.) (Poaceae) [35, 39]

- Indet.: Poaceae [39]

117. Metopolophium (Metopolophium) lacheni Agarwala, Mondal \& Raychaudhuri,

=Metopolophium (Metopolophium) darjilingense lacheni Agarwala, Mondal \& Raychaudhuri, 1982 [127]

= Metopolophium (Microlophium) darjeelingense lacheni Agarwala, Mondal \& Raychaudhuri, 1982

- Rubus sp. (Rosaceae) [125]

118. Metopolophium (Metopolophium) longicaudatum (David \& Hameed, 1975)

= Acyrthosiphon (Metopolophium) longicaudatum David \& Hameed, 1975 [71]

- Triticum sp. (Poaceae) [71, 207]

119. Metopolophium sp.

- Alcea rosea L. (Malvaceae) [71, 200]

- Rubus ellipticus Sm. (Rosaceae) [12]

- Rubus sp. (Rosaceae) [107]

- Rumex sp. (Polygonaceae) [69, 208]

- Triticum sp. (Poaceae) [70, 71]

120. Microlophium carnosum (Buckton, 1876)

= Metopolophium (Microlophium) carnosum (Buckton, 1876) [17]

= Microlophium evansi (Theobald, 1923) [121]

- Datura metel L. (=Datura fastuosa L.) (Solanaceae) [83]

- Urtica dioica L. (Urticaceae) [58, 72, 209]

- Urtica parviflora Roxb. (Urticaceae) [121]

- Urtica sp. (Urticaceae) [83, 144]

121. Microlophium rubiformosanum Takahashi, 1927

= Acyrthosiphon rubiformosanum (Takahashi, 1927) [105]

= Macrosiphum rubiformosanum Takahashi, 1927 [65]

- Potentilla nepalensis Hook (Rosaceae) [40]

- Rubus ellipticus Sm. (Rosaceae) [94, 105]

International J. Research Studies in Zoology (IJRSZ)

Page $\mid 57$ 
- Rubus rosifolius Sm. (=Rubus rosaefolia auct.) (Rosaceae) [65, 93]

- Urtica dioica L. (Urticaceae) [12, 179]

122. Microlophium sikkimensis Das, Bhattacharya, Ghosh \& Goswami, 2005

= Metopolophium (Microlophium) sikkimensis Das, Bhattacharya, Ghosh \& Goswami, 2005

- Euphorbia sp. (Euphorbiaceae) [210]

123. Micromyzella judenkoi (Carver, 1965)

= Micromyzus judenkoi Carver, 1965 [211]

- Adiantum caudatum L. (Pteridaceae) [13]

- Asplenium sp. (Aspleniaceae) [20, 22]

- Athyrium sp. (Dryopteridaceae) [20, 22]

- Cheilanthes argentea (S.G. Gmel.) G. Kunze (Pteridaceae) [39]

- Cheilanthes decomposita Willd. (=Cheilanthes compositor auct. nonn.) (Pteridaceae) [20, 165]

- Cheilanthes sp. (Pteridaceae) [24, 166]

- Unidentified plant [211]

124. Micromyzodium dasi Verma, 1969 (1970)

- Adiantum caudatum L. (Pteridaceae) [96]

- Cheilanthes sp. (Pteridaceae) [13, 96]

125. Micromyzodium filicium David, 1958

- Adiantum caudatum L. (Pteridaceae) [13]

- Adiantum cuneipinnulum N.C.Nair \& S.R.Ghosh (Pteridaceae) [180]

- Adiantum pseudotinctum Hier. (=Adiantum tinctum auct. nonn.) (Pteridaceae) [24,166]

- Asplenium trichomanes L. (Aspleniaceae) [24, 71, 94, 166]

- Cheilanthes marginata Kunth (Pteridaceae) [147]

- Cheilanthes sp. (Pteridaceae) [13]

- Didymocarpus pedicellata R. Br. (Gesneriaceae) [147]

- Dryopteris cochleata (D. Don) C. Chr. (Dryopteridaceae) [13]

- Dryopteris sp. (Dryopteridaceae) [13]

- Eriosorus peruviana L. (Pteridaceae) [103, 166]

- Geranium divaricatum Ehrh. (Geraniaceae) [71]

- Geranium lucidum L. (Geraniaceae) [71, 184]

- Nephrolepis sp. (Nephrolepidaceae) [24, 103, 166]

- Oxalis latifolia Kunth (Oxalidaceae ) [71, 184]

- Pityrogramma chrysophylla (Sw.) Link (=Pityrogramma chrysophila auct.nonn.) (Pteridaceae) $[20,22]$

- Pityrogramma peruviana (Desv. Maxon) (Pteridaceae) [24]

- Polypodium sp. (Polypodiaceae) [24, 103, 166]

- Pteris cretica L. (Pteridaceae) [24, 166]

- Pteris longifolia L. (Pteridaceae) [13]

- Punica granatum L. (Punicaceae) [71]

- Streptocarpus sp. (Gesneriaceae) [24, 166]

- Thelypteris (=Lastrea) sp. (Thelypteridaceae ) [24, 166]

126. Micromyzodium levipes (Basu \& Raychaudhuri, 1974)

= Eomyzus levipes Basu \& Raychaudhuri, 1974

- Eupatorium odoratum L. (Asteraceae) [20, 45]

127. Micromyzodium strobilanthi Ghosh, 1970 (1971)

- Ruellia tuberosa L. (Acanthaceae) [107]

- Strobilanthes dalhousianus Clarke (Acanthaceae) [71, 212]

- Strobilanthes sp. (Acanthaceae) [147]

128. Micromyzodium sp.

- Ailanthus sp. (Simaroubaceae) [71]

- Polygonum sp. (Polygonaceae) [93] 
129. Micromyzus kalimpongensis Basu, 1967 (1968)

- Amomum subulatum Roxb. (Zingiberaceae) [68]

- Canna sp. (Cannaceae) [68]

- Curcuma longa L. (Zingiberaceae) [20, 22, 80, 98]

- Elettaria cardamomum (L.) Maton (Zingiberaceae) [26, 37]

- Hedychium coronarium J. Koeing (Zingiberaceae) [20, 22]

- Hedychium sp. (Zingiberaceae) [26]

- Zingiber sp. (Zingiberaceae) [26]

- Undet.: Orchidaceae) [26, 37]

130. Micromyzus mawphlangensis Ghosh, 1974

- Polypodium sp. (Polypodiaceae) [18, 20, 24, 37]

131. Micromyzus niger van der Goot, 1917

= Micromyzus nigrum van der Goot, 1917 [17]

- Cheilanthes farinosa Kaulf. (Pteridaceae) [168]

- Eriosorus sp. (Pteridaceae) [26]

132. Micromyzus sp.

- Corylopsis sp. (Hamamelidaceae) [29]

- Elettaria cardamomum (L.) Maton (Zingiberaceae) [213]

- Hedychium sp. (Zingiberaceae) [65]

- Symplocos sp. (Symplocaceae) [20, 22, 37]

133. Myzakkaia kuwanais (Ghosh, Basu \& Raychaudhuri, 1970)

= Vesiculaphis kuwanais Ghosh, Basu \& Raychaudhuri, 1970

= Vesiculaphis kuwanis Ghosh, Basu \& Raychaudhuri, 1970 [214]

- Lyonia ovalifolia (Wall.) Drude (Ericaceae) [22]

134. Myzakkaia polygonicola Basu, 1969

- Persicaria nepalensis (Meisn.) H. Gross (=Polygonum nepalense Meisn.) (Polygonaceae) [13]

- Polygonum alatum Buch.-Ham. ex D. Don (Polygonaceae) [71]

- Polygonum runcinatum Buch.-Ham. ex D. Don (Polygonaceae) [20]

135. Myzakkaia verbasci (Chawdhuri, Basu, Chakrabarti \& Raychaudhuri, 1969)

= Myzakkaia himalayensis Basu, 1969

= Vesiculaphis verbasci Chawdhuri, Basu, Chakrabarti \& Raychaudhuri, 1969 [20, 22, 26, 39, 71, 80, 98, 109, 115, 201, 214]

- Amaranthus sp. (Amaranthaceae) [180]

- Artemisia sp. (Asteraceae) [20, 22]

- Asplenium sp. (Aspleniaceae) [71]

- Cynodon dactylon (L.) Pers. (Poaceae) [22]

- Ipomoea lobata (Cerv.) Thell. (Convolvulaceae) [20, 22,]

- Montanoa bipinnatifida (Kunth) K. Koch (Asteraceae) [22]

- Nepeta cataria L. (=Nepeta leucophylla Benth.) (Lamiaceae) [12]

- Passiflora sp. (Passifloraceae) [20, 22]

- Persicaria chinensis (L.) H. Gross (=Polygonum chinense L.) (Polygonaceae) [20, 22, 167]

- Photinia sp. (Rosaceae) [20, 22, 167]

- Polygonum alatum Buch.-Ham. ex D. Don (Polygonaceae) [20, 29, 71, 129, 167]

- Polygonum flaccidum Roxb. (=Polygonum serrulatum Lagasca) (Polygonaceae) [20, 22]

- Polygonum runcinatum Buch.-Ham. ex D. Don (Polygonaceae) [20, 22, 26, 167]

- Polygonum sp. (Polygonaceae) [20, 22, 26, 80, 98, 214]

- Rhamnus sp. (Rhamnaceae) [107]

- Rubia cordifolia L. (Rubiaceae) [71]

- Verbascum thapsus L. (Scrophulariaceae) [20, 22, 71, 120, 129]

- Viburnum sp. (Adoxaceae) [71]

- Indet.: Rosaceae [39] 
136. Myzaphis avariolosa David, Rajasingh \& Narayanan, 1970 (1971)

- Rosa macrophylla Lindl.(Rosaceae) [71]

137. Myzaphis rosarum (Kaltenbach, 1843)

= Aphis rossrum Kaltenbach, 1843 (misspelling for Aphis rosarum Kaltenbach, 1843) [107]

- Cornus oblonga Wall. (Cornaceae) [39]

- Rosa macrophylla Lindl. (Rosaceae) [39]

- Rosa moschata Herrm. (Rosaceae) [39]

- Rosa sp. (Rosaceae) [26, 28, 61, 71, 107, 147, 190]

- Rosa webbiana Wall. ex Royle (Rosaceae) [209]

138. Myzaphis turanica Nevsky, 1929

- Rosa brunonii Lindl. (Rosaceae) [72]

- Rosa macrophylla Lindl. (Rosaceae) [144]

- Rosa sp. (Rosaceae) [39]

139. Myzus (Myzus) brevisiphon Basu, 1969

= Myzus brevisiphon Basu, 1969

- Cissampelos pereira L. (Menispermaceae) [22, 39]

- Persicaria capitata (Buch.-Ham. ex D. Don) H. Gross (=Polygonum capitatum Buch.-Ham. ex D. Don) (Polygonaceae) [20, 22, 39- 41]

- Polygonum alatum Buch.-Ham. ex D. Don (Polygonaceae) [26]

- Polygonum sp. (Polygonaceae) [18, 26, 37]

- Rhododendron campanulatum D. Don (Ericaceae) [22]

- Rhododendron campylocarpum Hook.f. (Ericaceae) [37, 216]

140. Myzus (Myzus) cerasi cerasi (Fabricius, 1775)

= Myzus (Myzus) cerasi (Fabricius, 1775) [216]

= Myzus cerasi (Fabricius, 1775) [17]

= Myzus tsaengi Tao (misspelled for Myzus tsengi Tao)* [17, 170, 217]

- Artemisia sp. (Asteraceae) [22, 170, 216]

- Artemisia vulgaris L. (Asteraceae) [22, 170, 216]

- Cardamine sp. (Brassicaceae) [218]

- Crataegus sp. (Rosaceae) [20, 22]

- Galium asperifolium Wall. ex Roxb. (Rubiaceae) [13]

- Galium mollugo L. (Rubiaceae) [13]

- Phlox dummondii Hook. (Polemoniaceae) [219]

- Polygonum sp. (Polygonaceae) [26]

- Prunus cerasoides D. Don (Rosaceae) [20, 22, 39, 35, 216]

- Prunus cornuta (Wall. ex Royle) Steud. (Rosaceae) [20, 22, 216]

- Prunus persica (L.) Batsch (Rosaceae) [220]

- Prunus sp. (Rosaceae) [221]

- Rubia cordifolia L. (Rubiaceae) [20, 22, 35, 71, 216]

- Sambucus javanica Reinw. ex Blume (Adoxaceae) [20, 165, 170, 216]

- Spiraea sp. (Rosaceae) [222]

141. Myzus (Myzus) cerasi umefoliae (Shinji, 1924)

= Myzus umefoliae (Shinji, 1924) [17]

- Galium aparine L. (Rubiaceae) [20, 22, 69-71, 216]

- Galium mollugo L. (Rubiaceae) [13]

- Rubia cordifolia L. (Rubiaceae) [20, 22, 39, 140, 216]

- Smilax sp. (Smilacaceae) [204]

142. Myzus (Myzus) cornutus Medda \& Chakrabarti, 1986

- Prunus cornuta (Wall. ex Royle) Steud. (Rosaceae) [54, 56, 63]

143. Myzus (Myzus) dycei Carver, 1961

- Artemisia vulgaris L. (Asteraceae) [83]

- Digitalis purpurea L. (Plantaginaceae) [20, 22, 216] 
- Digitalis sp. (Plantaginaceae (=Scrophulariaceae)) [20, 22, 80, 216]

- Glycine max (L.) Merr. (Fabaceae) [20, 22, 26, 199, 216]

- Laportea sp. (=Fleurya sp.) (Urticaceae) [20, 22, 216]

- Nicotiana plumbaginifolia Viv. (Solanaceae) [194]

- Polygonum sp. (Polygonaceae) [20, 22, 98, 165, 216]

- Prunus cornuta (Wall. ex Royle) Steud. (Rosaceae) [13]

- Raphanus sativus L. (Brassicaceae) [30]

- Urtica dioica L. (Urticaceae) [20, 22, 147, 216]

- Urtica parviflora Roxb. (Urticaceae) [20, 22,26, 216]

- Urtica sp. (Urticaceae) [20, 22, 83, 107, 216]

- Valeriana wallichii De Candolle (Valerianaceae) [20, 22, 216]

- Undet.: Cucurbitaceae [80, 216]; Urticaceae [71, 109]

144. Myzus (Myzus) filicis Basu, 1969

- Undet.: Fern [20, 22, 24, 166, 167, 215, 216]

145. Myzus (Myzus) formosanus Takahashi, 1923

= Myzus leptotrichus David, Rajasingh \& Narayanan, 1972

- Impatiens balsamina L. (Balsaminaceae) [51, 57]

- Impatiens sp. (Balsaminaceae) [12, 221]

- Persicaria barbata (L.) H. Hara (=Polygonum barbatum L.) (Polygonaceae) [20, 216]

- Persicaria capitata (Buch.-Ham. ex D. Don) H. Gross (=Polygonum capitatum Buch.-Ham. ex D. Don) (Polygonaceae) [13, 139]

- Polygonum runcinatum Buch.-Ham. ex D. Don (Polygonaceae) [20, 22, 216]

146. Myzus (Myzus) hemerocallis Takahashi, 1921

- Agapanthus africanus (L.) Hoffmanns. (=A. umbellatus L.'Hér) (Agapanthaceae) [59, 138, 216]

- Hemerocallis lilioasphodelus L. (=Hemerocallis flava (L.)) (Hemerocallidaceae) [ 59, 138, 216]

147. Myzus (Myzus) indicus Basu \& Raychaudhuri, 1976

= Myzus indicum Basu \& Raychaudhuri, 1976 [17]

- Aneilema sp. (Commelinaceae) [13, 96]

- Boehmeria sp. (Urticaceae) [20, 22, 216]

148. Myzus (Myzus) kalimpongensis (Ghosh, Basu \& Raychaudhuri, 1976)

= Myzus kalimpongensis (Ghosh, Basu \& Raychaudhuri, 1976) [17]

= Vesiculaphis kalimpongensis Ghosh, Basu \& Raychaudhuri, 1976 [36, 98, 118]

- Artemisia sp. (Asteraceae) [20, 98, 214]

- Leonurus sibiricus L. (Lamiaceae) [20, 214]

149. Myzus (Myzus) lefroyi Basu \& Raychaudhuri, 1976

- Lindenbergia indica (L.) Volke (Plantaginaceae) [13]

- Undet.: Fern [22, 216]

150. Myzus (Myzus) maculocorpus Basu \& Raychaudhuri, 1976

- Unidentified plants [20, 22, 167, 216]

151. Myzus (Myzus) manoji Basu \& Raychaudhuri, 1976

- Hedera nepalensis K. Koch (Araliaceae) [223]

- Unidentified plant [167]

152. Myzus (Myzus) meghalayensis Basu \& Raychaudhuri, 1976

- Unidentified plants [20, 22, 216]

153. Myzus (Myzus) mumecola (Matsumura, 1917)

- Prunus cornuta (Wall. ex Royle) Steud. (Rosaceae) [22, 69, 71, 114, 216, 221]

- Prunus dulcis (Mill.) D.A. Webb. (=Prunus amygdalus Batsc) (Rosaceae) [224]

- Prunus sp. (Rosaceae) [54, 139]

- Rubia cordifolia L. (Rubiaceae) [58] 
154. Myzus (Myzus) obtusirostris David, Narayanan \& Rajasingh, 1971

- Apluda mutica L. (Poaceae) [107, 147]

- Chrysopogon sp. (Poaceae) [13]

- Lindera sp. (Lauraceae) [20, 22, 135, 216]

- Paspalum sp. (Poaceae) [83]

- Poa sp. (Poaceae) [225]

- Indet.: Poaceae [71]

155. Myzus (Myzus) ornatus Laing, 1932

- Achyranthes sp. (Amaranthaceae) [22]

- Ageratum conyzoides L. (Asteraceae) [22, 226]

- Ajuga brachystemon Maxim. (Lamiaceae) [13]

- Ajuga parviflora Benth. (Lamiaceae) [13]

- Alnus sp. (Betulaceae) [22]

- Alternanthera philoxeroides (Mart.) Griseb. (Amaranthaceae) [22]

- Amaranthus sp. (Amaranthaceae) [22, 226]

- Cyperus rotundus L. (Cyperaceae) [106]

- Nicotiana plumbaginifolia Viv. (Solanaceae) [106]

- Antirrhinum majus L. (Plantaginaceae) [26]

- Ardisia sp. (Myrsinaceae) [22]

- Artemisia vulgaris L. (Asteraceae) [22, 67]

- Aster sp. (Asteraceae) [180]

- Begonia rex Putz. (Begoniaceae) [22]

- Begonia sp. (Begoniaceae) [39]

- Bidens biternata (Lour.) Merr. \& Sherff (Asteraceae) [67]

- Bidens pilosa L. (Asteraceae) [22, 226]

- Blumea lacera (Burm.f.) DC. (Asteraceae) [22]

- Boehmeria nivea (L.) Gaudich var. tenacissima (Gaudich) Miq. (=Boehmeria calladissima auct. nonn.) As appeared in DNR (Urticaceae) [22]

- Boehmeria sp. (Urticaceae) [22]

- Bougainvillea spectabilis Willd. (Nyctaginaceae) [22]

- Brassica napus L. (Brassicaceae) [22]

- Brassica oleracea L. (Brassicaceae) [22]

- Buddleja sp. (Scrophulariaceae) [22]

- Calamintha sp. (Lamiaceae) [22]

- Callicarpa sp. (Verbenaceae) [22]

- Canna indica L. (=Canna orientalis Bouche) (Cannaceae) [22, 226]

- Cardamine hirsuta L. (Brassicaceae) [22]

- Cestrum fasciculatum (Schltdl.) Miers (Solanaceae) [22]

- Chenopodium album L. (Chenopodiaceae) [22]

- Chenopodium sp. (Chenopodiaceae) [226]

- Chrysanthemum indicum L. (Asteraceae) [22]

- Cineraria sp. (Asteraceae) [22, 59, 101, 138]

- Clerodendrum chinense (Osbeck) Mabb. (=Clerodendrum fragrens Willd.) (Lamiaceae) [98]

- Clerodendrum sp. (Lamiaceae) [22]

- Clinopodium umbrosum (M. Bieb.) K. Koch (Lamiaceae) [227]

- Cnicus sp. (Asteraceae) [22, 226]

- Commelina bengalensis L. (Commelinaceae) [22, 226]

- Conyza japonica (Thunb.) DC. (Asteraceae) [22, 147]

- Coriandrum sativum L. (Apiaceae) [22]

- Cosmos sp. (Asteraceae) [22, 226]

- Cotula sp. (Asteraceae) [22]

- Cucurbita maxima Duchesne (Cucurbitaceae) [22] 
- Cucurbita sp. (Cucurbitaceae) [67]

- Cynoglossum wallichii G. Don (Boraginaceae) [22]

- Dahlia sp. (Asteraceae) [22, 26]

- Datura metel L. (=Datura fastuosa L.) (Solanaceae) [22]

- Dendrobium longicornu Lindl. (Orchidaceae) [22]

- Dichrocephala integrifolia (L.f.) Kuntze (=Dichrocephala latifolia (Pers.) DC.) (Asteraceae) [22]

- Dioscorea sp. (Dioscoreaceae) [13]

- Duranta erecta L. (=Duranta plumieri Jacq.; =Duranta repens L.) (Verbenaceae) [22, 67, 103, 228, 229]

- Duranta sp. (Verbenaceae) $[19,67]$

- Elsholtzia album ? (Lamiaceae) [22]

- Elsholtzia blanda (Benth.) Benth. (Lamiaceae) [22]

- Elsholtzia fruticosa (D. Don) Rehder (Lamiaceae) [13]

- Erigeron sp. (Asteraceae) [22, 226]

- Eupatorium adenophorum Spreng. (Asteraceae) [67]

- Eupatorium odoratum L. (Asteraceae) [22, 226]

- Eupatorium sp. (Asteraceae) [67]

- Eupatorium wallichii DC. (Asteraceae) [22]

- Fagopyrum sp. (Polygonaceae) [22, 226]

- Ficus sp. (Moraceae) [22]

- Fragaria nilgerrensis Schltdl. ex J. Gay (Rosaceae) [37, 39, 41, 216]

- Fragaria sp. (Rosaceae) [22]

- Fuchsia sp. (Onagraceae) [22, 26, 216]

- Gaillardia sp. (Asteraceae) [39]

- Galium mollugo L. (Rubiaceae) [39]

- Galium sp. (Rubiaceae) [22]

- Galium verum L. (Rubiaceae) [107]

- Gentiana sp. (Gentianaceae) [22]

- Geranium ocellatum Cambess. (Geraniaceae) [39]

- Geranium sp. (Geraniaceae) [22]

- Gladiolus sp. (Iridaceae) [22]

- Gynura nepalensis DC. (Asteraceae) [22]

- Gynura sp. (Asteraceae) [226]

- Helianthus annuus L. (Asteraceae) [22]

- Hibiscus mutabilis L. (Malvaceae) [22]

- Hibiscus rosa-sinensis L. (Malvaceae) [22]

- Hydrocotyle sp. (Araliaceae (=Hydrocotylaceae)) [22]

- Hypochaeris radicata L. (Asteraceae) [22]

- Iberis amara L. (Brassicaceae) [22]

- Iberis sp. (Brassicaceae) [39]

- Inula cappa (Buch.-Ham. Ex D. Don) DC. (Asteraceae) [22]

- Ipomoea hederacea Jacq. (Convolvulaceae) [22]

- Ipomoea lobata (Cerv.) Thell. (Convolvulaceae) [22]

- Iris domestica Goldblatt. \& Mabb. (= Belamcanda chinensis (L.) DC.) (Iridaceae) [98]

- Isodon rugosus (Wall ex Benth.) Codd (=Plectranthus rugosus Wall ex Benth.) (Lamiaceae) [39]

- Jacaranda acutifolia Bonpl. (Bignoniaceae) [22]

- Justicia simplex D. Don (Acanthaceae) [180]

- Lablab purpureus (L.) Sweet ssp. purpureus (=Dolichos lablab L.) (Fabaceae) [22]

- Lactuca sativa L. (Asteraceae) [71]

- Lagerstroemia indica L. (Lythraceae) [22]

- Lantana camara L. (Verbenaceae) [22, 67, 226] 
- Leucosceptrum cannum Sm. (Lamiaceae) [67]

- Lindenbergia sp. (Plantaginaceae) [71]

- Lonicera macrantha (D. Don). Don) Spreng. (Caprifoliaceae) [22]

- Lycopersicon esculentum Mill. (Solanaceae) [26]

- Malvaviscus conzattii Grenm. (=Malvaviscus conzonthiae auct.) (Malvaceae) [67]

- Mentha sp. (Lamiaceae) [22]

- Mirabilis jalapa L. (Nyctaginaceae) [22]

- Montanoa bipinnatifida (Kunth) K. Koch (Asteraceae) [22]

- Morus sp. (Moraceae) [22]

- Nageia nagi (Thunb.) Kuntz. (=Myrica nagi Thunb.) (Myricaceae) [22]

- Nicotiana sp. (Solanaceae) [39, 61]

- Nicotiana tabacum L. (Solanaceae ) [22, 39]

- Ocimum tenuiflorum L. (=Ocimum sanctum L.) (Lamiaceae) [22]

- Osbeckia capitata Bentm. Ex Naudin (Melastomaceae) [22]

- Osbeckia chinensis L. (=Osbechia sinensis auct. non.) (Melastomaceae) [226]

- Oxalis corniculata L. (Oxalidaceae) [22]

- Oxalis triflora ? (Oxalidaceae) [22]

- Passiflora foetida L. (Passifloraceae) [22]

- Pedicularis sp. (Orobanchaceae) [13]

- Persicaria chinensis (L.) H. Gross (=Polygonum chinense L.) (Polygonaceae) [22]

- Plantago major L. (Plantaginaceae) [22]

- Polygonum alatum Buch.-Ham. ex D. Don (Polygonaceae) [22]

- Polygonum flaccidum Roxb. (=Polygonum serrulatum Lagasca) (Polygonaceae) [22]

- Polygonum sp. (Polygonaceae) [71, 226]

- Potalia sp. (Gentianaceae) [22]

- Prunella vulgaris L. (Lamiaceae) [22]

- Prunus cerasoides D. Don (=Prunus puddum (Rox. Ex Ser.)) (Rosaceae) [22, 101]

- Prunus cerasus L. (Rosaceae) [22]

- Prunus napaulensis (Ser.) Steud. (Rosaceae) [22]

- Prunus sp. (Rosaceae) [147]

- Pseudognaphalium luteoalbum (L.) Hill. \& Burtt (=Gnaphalium luteoalbum L.) (Asteraceae) $[22,226]$

- Punica granatum L. (Punicaceae) [22]

- Pyrus communis L. (Rosaceae) [22]

- Raphanus sativus L. (Brassicaceae) [22]

- Rhamnus napalensis (Wall.) M.A. Lawson (Rhamnaceae) [107]

- Rhamnus sp. (Rhamnaceae) [107]

- Rhododendron arboreum Sm. (Ericaceae) [22]

- Richardia pilosa L. (Rubiaceae) [22]

- Ricinus communis L. (Euphorbiaceae) [22]

- Rorippa indica (L.) Hiern (=Nasturtium indicum (L.) DC.) (Brassicaceae) [22]

- Rosa sp. (Rosaceae) [22]

- Rubia cordifolia L. (Rubiaceae) [22]

- Rubus ellipticus Sm. (Rosaceae) [22, 226]

- Rubus rosifolius $\mathrm{Sm}$. (=Rubus rosaefolia auct.) (Rosaceae) [22]

- Rumex acetosella L. (Polygonaceae) [101]

- Rumex nepalensis Spreng. (Polygonaceae) [22, 71, 109]

- Salvia sp. (Lamiaceae) [69, 146]

- Sambucus javanica Reinw. ex Blume (Adoxaceae (=Sambucaceae)) [22]

- Schima wallichii (DC.) Korth. (Theaceae) [22]

- Scutellaria discolor Colebr. (Lamiaceae) [22]

- Scutellaria scandens Buch.-Ham. ex D. Don (Lamiaceae) [13]

- Senebiera pinnatifida DC. (Brassicaceae) [22]

- Senecio sp. (Asteraceae) [109, 138] 
- Solanum aurantiacum Rojas (Solanaceae) [22]

- Solanum betaceum Cav. (=Cyphomandra betacea (Cav.) Sendtn.) (Solanaceae) [22]

- Solanum clavatum Rusby (Solanaceae) [22]

- Solanum melongena L. (Solanaceae) [22]

- Solanum nigrum L. (Solanaceae) [22]

- Solanum sp. (Solanaceae) [39, 61, 226]

- Solanum tuberosum L. (Solanaceae) [69, 71, 101, 187]

- Sonchus arvensis L. (Asteraceae) [226]

- Sonchus brachyotus DC. (Asteraceae) [39]

- Sonchus sp. (Asteraceae) [22, 104]

- Sorbaria sorbifolia (L.) Braun (=Spiraea sorbifolia L.) (Rosaceae) [22, 39, 69, 71, 120, 129]

- Spinacia oleracea L. (Chenopodiaceae) [22]

- Spiraea callosa Thunb. Ex Murr. (Rosaceae) [22]

- Spiraea sp. (Rosaceae) [61]

- Stachys sericea Wall. ex Benth. (Lamiaceae) [40]

- Stellaria crispate Wall. ex D. Don (Caryophyliaceae) [22]

- Stellaria media (L.) Vill. (Caryophyliaceae) [22]

- Strobilanthes sp. (Acanthaceae) [22]

- Tagetes patula L. (Asteraceae) [22]

- Thunbergia coccinea Wall. ex D. Don (Acanthaceae (=Thunberigiaceae)) [22]

- Tibouchina semidecandra (Schrank \& Mart. Ex DC.) Cogn. (Melastomaceae) [22]

- Tridax procumbens L. (Asteraceae) [22]

- Tropaeolum majus L. (Tropaeolaceae) [22]

- Urena lobata L. (Malvaceae) [22]

- Urena sp. (Malvaceae) [83]

- Valeriana wallichii De Candolle (Valerianaceae) [22]

- Verbena sp. (Verbenaceae) [22]

- Viburnum foetidum Wall. (Adoxaceae (=Caprifoliaceae)) [22]

- Vicia faba L. (Fabaceae) [22]

-Vigna unguiculata (L.) Walp. ssp. cylindrica (L.) Verdc. (=Vigna catjang (Burm.f.) Walp.)

(Fabaceae) [22]

- Viola sp. (Violaceae) [71]

- Viola tricolor L. (Violaceae) [22]

- Wendlandia sp. (Rubiaceae) [22]

- Zanthoxylum sp. (Rutaceae) [22]

- Zinnia elegans Jacq. (Asteraceae) [22]

- Undet.: Asteraceae [180, 199]; Lamiaceae [64]; Poaceae [71, 107]; Vitidaceae [83]

156. Myzus (Myzus) siegesbeckicola Strand, 1929

- Artemisia sp. (Asteraceae) [180]

- Aster sp. (Asteraceae) [180]

- Eupatorium odoratum L. (Asteraceae) [20, 22, 216]

- Helianthus annuus L. (Asteraceae) [180]

- Lantana camara L. (Verbenaceae) [180]

- Leucas sp. (Lamiaceae) [180]

- Montanoa bipinnatifida (Kunth) K. Koch (Asteraceae) [20, 22, 216]

- Perilla sp. (Lamiaceae) [20, 22, 216]

- Prunus cerasus L. (Rosaceae) [98, 230]

- Siegesbeckia orientalis L. (Asteraceae) [20, 22, 147, 26, 216]

- Tecoma capensis (Thunb.) Lindl. (Bignoniaceae) [180]

- Urtica dioica L. (Urticaceae) [231]

- Verbena sp. (Verbenaceae) [180] 
157. Myzus (Myzus) sorbi Bhattacharya \& Chakrabarti, 1982

- Sorbaria tomentosa (Lindl.) Rehder (Rosaceae) [48, 63]

- Sorbus sp. (Rosaceae) [12]

158. Myzus (Myzus) varians Davidson, 1912

- Prunus armeniaca L. (Rosaceae) [83, 232]

- Prunus cerasus L. (Rosaceae) [83, 232]

- Prunus domestica L. (Rosaceae) [83, 232]

- Prunus dulcis (Mill.) D.A. Webb. (=Prunus amygdalus Batsch) (Rosaceae) [233]

159. Myzus (Nectarosiphon) ajari Sathe \& Jadhav, 2008§

- Nicotiana tabacum Linn. (Solanaceae) [137]

160. Myzus (Nectarosiphon) persicae (Sulzer, 1776)

= Myzus persicae (Sulzer, 1776) [17]

= Rhopalosiphum dianthi Schrank, 1801 [193]

Myzus (Nectarosiphon) persicae is a highly polyphagous aphid species and is now considered to consist of distinct phenotypes and genotypes, both holocyclic and anholocyclic, that vary with respect to their ability to reproduce and food preferences on different host plants. Its diversity of food plant in India has already been catalogued recently [3] that includes plants belonging to 293 species under 64 plant families. There are 14 plant families where more than 5 species of plants were infested by it in India, viz. Asteraceae (44 plant species), Solanaceae (37 plant species), Brassicaceae (27 plant species), Fabaceae (18 plant species), Rosaceae (13 plant species), Malvaceae (11 plant species), Convolvulaceae (11 plant species), Amaranthaceae (10 plant species), Polygonaceae (8 plant species), Caryophyllaceae, Plantaginaceae, Poaceae (7 plant species each), Cucurbitaceae (6 plant species), and Euphorbiaceae (5 plant species).

161. Myzus (Nectarosiphon) terminalae Sathe \& Jadhav, 2008§

- Terminalia tomentosa Mart.ex Eichl. (Combretaceae) [137]

162. Myzus (Sciamyzus) ascalonicus Doncaster, 1946

- Calamintha sp. (Lamiaceae) [20, 22, 170, 216]

- Cerastium glomeratum Thuill (Caryophyliaceae) [170]

- Chrysanthemum sp. (Asteraceae) [20, 22, 216]

- Brassica spp. [234]

- Fragaria sp. (Rosaceae) [69, 71, 120, 216]

- Hypochaeris radicata L. (Asteraceae) [20, 22, 170, 216]

- Isodon coesta (Buch.-Ham. ex D. Don ) (=Plectranthus coetsa Buch.-Ham. ex D. Don) (Lamiaceae) [20, 22, 69, 120, 216]

- Papaver somniferum L. (Papaveraceae) [170]

- Primula sp. (Primulaceae) [20, 22, 216]

- Pseudognaphalium luteoalbum (L.) Hill. \& Burtt (=Gnaphalium luteoalbum L.) (Asteraceae) [20, 22, 216]

- Richardia pilosa L. (Rubiaceae) [20, 22, 170, 216]

- Senebiera pinnatifida DC. (Brassicaceae) [170]

- Stellaria crispata Wall. ex D. Don (Caryophyliaceae) [20, 22, 216]

- Stellaria media (L.) Vill. (Caryophyliaceae) [20, 22, 170, 216]

\section{CONCLUSION}

Food plants of 38 genera and 148 species of aphids belonging to the tribe Macrosiphini (Elatobium to Myzus) are catalogued in this contribution. These aphids feed on 416 plant species belonging to 98 families. Asteraceae (62 species) followed by Rosaceae (41 species), Brassicaceae (26 species), Lamiaceae (23 species), Fabaceae (18 species), Pteridaceae (14 species), and Solanaceae (13 species) are most suffered plant families. Among them, several species are crops of agricultural and horticultural importance. 


\section{REFERENCES}

[1] Favret, C., Aphid Species File. Version 5.0/5.0. <http,//Aphid.SpeciesFile.org> (accessed on January 2, 2017).

[2] Chan, C.K., Forbes, A.R. and Raworth, D.A., Aphid transmitted viruses and the vectors of the world. Agriculture Canada, Ottawa, ON, Tech. Bull. 1991-3E, pp. 216 (1991).

[3] Tiwari, A.K. and Singh, R., Effect of host plants on the morphology of green peach aphid, Myzus persicae (Sulzer) (Homoptera, Aphididae), Internat. J. Zool. Investig. 2(1), 133-146 (2016).

[4] Nieto Nafria, J.M. and Favret, C., Update to the Registers of family-group and genusgroup taxa of Aphidoidea (Hemiptera, Sternorrhyncha). Boln. Asoc. esp. Ent. 38 (1-2), 1-23 (2014).

[5] Heie, O.E. and Wegierek, P., Diagnoses of the higher taxa of Aphidomorpha (Hemiptera, Sternorrhyncha). Redia 92, 261-269 (2009).

[6] Nieto Nafría, J.M. and Favret, C. (Eds.), Registers of Family-Group and Genus-Group Taxa of Aphidoidea (Hemiptera Sternorrhyncha), Universidad de León. León (Spain), pp. 466 (2011).

[7] Remaudiere, G. and Remaudiere, M., Catalogue of the World's Aphididae. Instut National de la Recherche Agronomique, Paris, pp. 473 (1997).

[8] Buga, S.V. and Stekolshchikov, A.V., Aphids of the tribe Macrosiphini (Insecta: Homoptera: Aphididae) in Belarus. Zoosyst. Rossica 21(1), 63-96 (2012).

[9] Singh, G. and Singh, R., Food plant records of Aphidini (Aphidinae, Aphididae, Hemiptera) in India. J. Ent. Zool. Stud. 5(1), submitted.

[10] Singh, G. and Singh, R., Updated checklist of food plants of Macrosiphini (Aphididae: Hemiptera) in India -1. Internat. J. Res. Stud.Zool. (IJRSZ), submitted.

[11] Agarwala, B.K. Pramanik, D.R. and Raychaudhuri D., Some new and little known macrosiphine aphids (Homoptera: Aphididae) from India. Akitu, N.S. 47, 1-7 (1982).

[12] Kar, I., Basu, G. and Khuda-Bukhsh, A.R., A Check-list of chromosomes in aphids (Homoptera: Aphididae) worked out in India along with the names and families of their host plants. Environ. and Ecol. 8(1), 414-428 (1990).

[13] Chakrabarti, S. and Sarkar, A., A supplement to the food-plant catalogue of Indian Aphididae. J. Aphidol. 15, 9-62 (2001).

[14] Chakrabarti, S. and Maity, S.P., A new genus and four new species of aphids (Homoptera: Aphididae) from Northwest Himalaya, India. Orient. Ins. 18, 195-212 (1984).

[15] Medda, P.K. Sarkar, A. and Chakrabarti, S., Willow infesting aphids (Homoptera: Aphididae) of India and adjacent countries. J. Aphidol. 11(1), 83-97 (1997).

[16] Chakrabarti, S. Biosystematics of gall aphid (Aphididae, Homoptera) of Western Himalaya. Proc. Indian Acad. Sci. 96(5), 561-572 (1987).

[17] Raychaudhuri, D.N. (Ed.), Food Plant Catalogue of Indian Aphididae. Graphic Printall, Culcutta (India). pp. 204 (1983).

[18] Ghosh, A.K. and Agarwala, B.K., A catalogue of aphidiid (Hymenoptera: Aphidiidae) parasites of aphids (Homoptera) of India. J. Bombay nat. Hist. Soc. 79, 125-134 (1985).

[19] Raychaudhuri, D.N. (Ed.), Aphids of North-East India and Bhutan. Zool. Soc., Calcutta, pp. 521 (1980).

[20] Ghosh, A.K. and Raychaudhuri, D.N., A note on Chaetomyzus, Ericolophium and Neoacyrlhosiphon, including a new subgenus Pseudoacyrthosiphon (Homoptera) from India. Orient. Ins. 3, 93-96 (1969).

[21] Raychaudhuri, D.N., Taxonomy of the aphids of the Eastern Himalayas. US P1 480 Project Tech. Rep., pp. 107 (1973).

[22] Chakrabarti, S. Mandal, A.K. and Raha, S., Rhododendron infesting aphids (Homoptera: Aphididae) of the Himalayas. Zool. J. Linn. Soc. 78, 349-362 (1983).

[23] Ghosh, A.K., Floral assemblage and faunal diversity in Aphidoidea (Homoptera: Aphididae) in Eastern India. Bull. zool. Surv. India 2, 171-176 (1980).

[24] Raychaudhuri, D.N. Poddar, S.C. and Raychaudhuri, D., Study of the genus Aphidius (Hymenoptera, Aphidiidae) of India. Entomon 7, 11-22 (1982). 
[25] Agarwala, B.K., Some aspects of aphid (Homoptera, Insecta) studies in Sikkim and Bhutan Ph. D. thesis, University of Calcutta, India, pp. 383 (1979).

[26] Agarwala, B.K., Dutta, S. and Raychaudhuri, D.N., An account of syrphid ( Diptera : Syrphididae) predators of aphids (Homoptera : Aphididae) available in Darjeeling district of West Bengal and Sikkim. Pranikee, $3: 15-21$ (1982).

[27] Agarwala, B.K. Ghosh, D. Das, S.K. Poddar, S.C. and Raychaudhuri, D.N., Parasites and predators of aphids (Homoptera, Aphididae) from India - V. New records of two aphidiid parasites, nine arachnid and one dipteran predators from India. Entomon 6, 233-238 (1981).

[28] Ghosh, A.K., Banerjee, H. and Raythaudhuri, D.N. (1971b). Studies on the aphids (Homoptera: Aphididae) from eastern India. 10. New species and further new records from Sikkim. Proc. zool. Soc. Calcutta 24, 99-111.

[29] Raha, S.K. Singh, T.K., Raychaudhuri, D. and Raychaudhuri, D.N., New records of aphids (Homoptera, Aphididae) from Manipur and Nagaland. Sci. and Cult. 43, 452-453 (1977).

[30] Ghosh, M.R. Ghosh, A.K. and Raychaudhuri, D.N., Studies on the aphids (Homoptera, Aphididae) from eastern India. III. New genus, new species and new records from north Bengal and Sikkim. Orient. Ins. 4, 377-393 (1970).

[31] Agarwala, B.K. and Mahapatra, S.K., A new species of Ericolophium Tao (Homoptera: Aphididae) from India. Entomon 11, 299-300 (1986).

[32] Ghosh, A.K., Cervaphidini (Homoptera: Aphididae) of the world. Orient. Ins. 16, 77-97 (1982).

[33] David, S.K. and Rajasingh, S.G., New records of aphids (Insecta, Homoptera) from Assam, India. Proc. zool. Soc. Calcutta, 22, 151-157 (1969).

[34] Chakrabarti, S., Ghosh, A.K. and Raychaudhuri, D.N., A new genus, a new species and further records of aphids (Homoptera : Aphididae) from the Kumaon Hills, Northwest Himalaya. Orient. Ins. 6, 387-400 (1972).

[35] Chakrabarti, S., Ghosh, A.K. and Raychaudhuri, D.N., A new genus, a new species and further records of aphids (Homoptera: Aphididae) from the Kumaon hills, northwest Himalaya, India. Orient. Ins. 6, 387-400 (1972).

[36] Basu, R.C., Ghosh, M.R. and Raychaudhuri, D.N., Studies on the aphids (Homoptera : Aphididae) from Eastern India. XXXIV. Two new genera, three new subgenera, one new species and some new records from northeast India. Entomon 1, 59-66 (1976).

[37] Basu, R.C. and Raychaudhuri, D.N., A study on the sexuales of aphids (Homoptera, Apididae) in India. Rec. Zool. Surv. India, Occ. Paper 18, 1-54 (1980).

[38] Ghosh, A.K., New aphids (Insecta: Homoptera) from North Bengal, India. Proc. zool. Soc. Calcutta 22, 121-127 (1969).

[39] Chakrabarti, S., Aphids of north western India with special reference to Kumaon range, Uttar Pradesh, Ph. D. thesis. University of Calcutta, India, pp. 435 (1972).

[40] Chakrabarti, S., Ghosh, A.K. and Raychaudhuri, D.N., On some undescribed morphs and new records of aphids (Homoptera, Aphididae) from Kumaon Himalaya, India. Curr. Sci. 41, 70-71 (1972).

[41] Chakrabarti, S. and Raychaudhuri, D.N., Aphids from Sundadhirga Valley Kumaon Himalaya, India. Orient. Ins. 9, 195-211 (1975).

[42] Ghosh, A.K., Notes on two new records of aphids (Homoptera : Aphididae) from India. Bull. Ent. 10, 107-109 (1969).

[43] Chakrabarti, S., A new genus and two new species of aphids (Homoptera : Aphididae) from northwest India. J. Linn. Soc. Zool. 62, 355-363 (1978).

[44] Ghosh, L.K., Aphids of NEFA, India (Homoptera: Aphididae). Sci. and Cult. 36, 562-563 (1970).

[45] Basu, R.C. and Raychaudhuri, D.N., Two new species of aphids (Homoptera : Aphididae) from eastern India. Orient. Ins. 8, 489-493 (1974).

[46] Chakrabarti, S. and Bhattacharya, D.K., New genus and species of aphids (Homoptera, Aphididae) from north-western Himalaya. Ann. Zool. 36(28), 539-547 (1982). 
[47] Maity, S.P., Bhattacharya, D.K. and Chakrabarti, S., Four new species of aphids (Homoptera: Aphididae) from Garhwal Himalaya, Uttar Pradesh, India. Ann. Zool. 36(26), 501-516 (1982).

[48] Maity, S.P. and Chakrabarti, S., Aphids (Homoptera : Aphididae) of north west India-III : records of new aphids from Garhwal Himalaya. Sci. and Cult. 45, 68-162 (1979).

[49] Medda, P.K. and Chakrabarti, S., Hitherto unknown alate morphs of two species of Eumyzus Shinji (Homoptera : Aphididae) from India with a key to alate viviparous females. Syst. Ent. 11, 107-110 (1986).

[50] Bhattacharya, D.K. Mandal, A.K. and Chakrabarti, S., New and hitherto not known species of aphids (Homoptera: Aphididae) producing leaf galls in the north west Himalayas, India. Entomon, 8, 13-17 (1983).

[51] Medda, P. and Chakrabarti, S., Two new aphid species from the western Himalaya and an account of the Pyrus-infesting generations of Melanaphis pahanensis (Homoptera : Aphidoidea : Aphididae). Entomol. Gener. 17(2), 139-146 (1992).

[52] Raychaudhuri, D. Singh, T.K. and Raychaudhuri, D.N., Some new and unknown aphids (Homoptera, Aphididae) from Manipur, northeast India. Entomon 9(1), 53-56 (1984).

[53] Medda, P.K., Saha, S. and Chakrabarti, S., Prunus- infesting aphids of North west Himalaya. Proc. $2^{\text {nd }}$ Nat. Symp. Recent trends in Aphidol. St., pp 29-38 (1986).

[54] Ghosh, D. Debnath, N. and Chakrabarti, S., Predators and parasites of aphids from northwest and western Himalaya. IV. Twelve species of heteropterans (Heteroptera: Insecta) from Garhwal and Kumaon ranges. Proc. zool. Soc. Calcutta 39, 15-19 (1986).

[55] Medda, P.K. and Chakrabarti, S., Two new aphids (Homoptera : Aphididae) forming leaf galls on Prunus spp. Entomon 11(3), 203-210 (1986).

[56] Bhattacharya, D.K., New and not known aphids (Homoptera : Aphididae) from Himachal Pradesh, India. J. Bom. Nat. Hist. Soc. 91, 117-120 (1994).

[57] Bhagat, R.C., New records of the aphids (Homoptera, Aphididae) from Kashmir (India). Sci. and Cult. 47, 134-136 (1981).

[58] Behura, B.K., Aphids of India. Survey of published information. Recent Adv. Zool. India 1961, 25-78 (1963).

[59] Ghulam-Ullah, Studies of Indian Aphididae - I, The Aphid fauna of Delhi. Indian J. Ent. 2, 1325 (1940).

[60] Chakrabarti, S. Ghosh, A.K. and Raychaudhuri, D.N., New records of aphids (Insecta: Homoptera) from Uttar Pradesh, India. Sci. and Cult. 37, 247-248 (1971).

[61] Joshi, S. and Poorani, J., Aphids of Karnataka. http,//www.aphidweb.com (accessed on 2 January, 2017).

[62] Chakrabarti, S. Sarkar, S. and Das, B.C., Aphid parasitoids (Hymenoptera: Braconidae: Aphidiinae) from western Himalaya. J. Aphidol. 16, 45-49 (2002).

[63] Basu, A.N., Further records of new and little known aphids (Homoptera) from West Bengal, India. Orient. Ins. 3, 355-371 (1969).

[64] Basu, R.C., Ghosh, A.K. and Raychaudhuri, D.N., A new species of Eutrichosiphum and notes on other new records of aphids (Insecta, Homoptera) from NEFA (Arunachal), Sci. and Cult. 38, 494-495 (1972).

[65] David, S.K., Notes on South Indian Aphids. IV. Aphidinae (continued). Indian J. Ent. 19, 289299 (1957).

[66] Ghosh, L.K., Additions to the aphid fauna of Bihar with the first record of an aphid sexuale (Homoptera, Aphididae). J. Bomb. nat. Hist. Soc. 73, 416-418 (1977).

[67] Basu, A.N., One new genus and seven new species of aphids from Darjeeling district, West Bengal (Homoptera: Aphididae). Bull. Ent. 8, 143-157 (1967).

[68] Bhalla, O.P. and Pawar, A.D., A survey of insect and non-insect pests of economic importance in Himachal Pradesh. Published by Department of Entomology and Zoology, College of Agriculture, Chambaghat, Solan (H.P.) (1980).

[69] Bindra, O.S. and Sekhon, S.S., Additional new records of aphids from the Kangra, Kulu and Lahaul valleys (India). Bull. Ent. 10, 156-157 (1969). 
[70] Ghosh, L.K., A study on the aphids (Homoptera, Aphididae) of Himachal Pradesh in NorthWest Himalaya, India, Ph. D. thesis, University of Calcutta, India, 360 pp. (1977).

[71] Rishi, N.D. (1975), Abst. Symp. 'Recent Trends in Aphidological Studies', Bhubaneswar, p. 52.

[72] Ghosh, A.K. and Verma, K.D., A new genus and new species of aphid (Homoptera: Aphididae) from northwest Himalaya. Orient. Ins. 7, 271-274 (1973).

[73] George, C.J., South Indian Aphididae. J. Asiatic. Soc. Bengal, (N.S.) 23, 1-12 (1927).

[74] Verma, K.D. and Das, S.M., The Aphididae of North-west India: with Special Reference to Aphids of Jammu and Kashmir State, New Delhi: Ashish Pub. House, 1992, pp. 171.

[75] Krishnamurthi, B., Aphididae of Mysore. II. J. Bombay nat. Hist. Soc. 34, 411-419 (1931).

[76] David, S.K., Taxonomic notes on six species of Mysore aphids described as new by Theobald in 1929. Indian J. Entomol. 18, 141-145 (1956).

[77] Verma, K.D., A new genus and two new species of aphids from northwest India (Homoptera: Aphididae). Bull. Ent. 12, 97-99 (1971).

[78] Basu, A.N. and Banerjee, S.N., Aphids of economic plants of West Bengal. Indian Agric. 2, 89112 (1958).

[79] Ghosh, A.K. Basu, R.C. and Raychaudhuri, D.N., Studies on the aphids (Homoptera,Aphididae) from eastern India. Orient. Ins. 4, 65-76 (1970).

[80] Ghosh, L.K., On a collection of aphids (Homoptera, Aphididae) from Uttar Pradesh, India. Sci. and Cult. 35, 493-494 (1969).

[81] Ghosh, L.K., A note on the preliminary survey of aphids (Homoptewra) from Bihar, India. Sci. and Cult. 36, 419-420 (1970).

[82] Raychaudhuri, D., Taxonomy and biology of aphids (Homoptera, Aphididae) of Manipur. Ph. D. thesis, University of Calcutta, India, pp. 308+XVI (1978).

[83] Verma, A.N., Khurana. A.D. and Bhanot, J.P., Aphids of Hissar (Haryana). Haryana Agric. Univ. J. Res. 5, 11-14 (1975).

[84] Ghosh, L.K., A Conspectus of Aphididae (Homoptera) of Himachal Pradesh in North-west Himalaya, India. Zool. Surv. India (Tech. Monog. No. 16, pp.1-282 (1986).

[85] George, C.J., The root sucking aphids of Coimbatore. J. Asiatic. Soc. Bengal (N.S.) 20, 307-310 (1925).

[86] David, S.K., Additional notes on some aphids in Madras State. Madras Agric. J. 43, 103-107 (1956).

[87] Theobald, F.V., Some aphidines from southern India with description of six new species. Entomologist 62, 177-181 and 196-201 (1929).

[88] Ahmad, M.E. and Singh, R., Records of Macrosiphini of northeastern Uttar Pradesh and its relationship with food plants and natural enemies. J. Aphidol. 9, 80-86 (1995).

[89] Singh, R. Upadhyay, B.S. Singh, D. Chaudhary, H.C., Aphids (Homoptera, Aphididae) and their parasitoids in north-eastern Uttar Pradesh. J. Aphidol. 13, 49-62 (1999).

[90] Bhattacharya, D.K., Notes on the Genus Hyalomyzus Richards (Homoptera : Aphididae) with description of a new species from India. Orient. Ins. 28, 199-203 (1994).

[91] Basu, R.C. Ghosh, A.K. and Raychaudhuri, D.N., Studies on the aphids (Homoptera, Aphididae) from eastern India. 18. Five new species and thirty new records from Assam. Proc. zool. Soc. Calcutta, 26, 89-101 (1973).

[92] Ghosh, A.K., Ghosh, M.R. and Raychaudhuri, D.N., Studies on the aphids (Homoptera: Aphididae) from eastern India. IX. One new genus, five new species, a new subspecies and further new records from the Darjeeling district, West Bengal. Orient. Ins. 5, 323-336 (1971).

[93] Ghosh, A.K., Ghosh, M.R. and Raychaudhuri, D.N., Studies on the aphids (Homoptera : Aphididae) from eastern India. IX. One new genus, five new species, a new subspecies and further new records from the Darjeeling district, West Bengal. Orient. Ins. 5, 323-336 (1971).

[94] Hille Ris Lambers, D. (1973), Notes on some oriental aphids, with descriptions of a new genus and four new species (Homoptera: Aphididae). Orient. Ins. 7, 239-259.

[95] Banerjee, P.K., Chakrabarti, S. and Chakrabarti, S. (1991), Host association and undescribed alate viviparous female of Matsumuraja capitophoroides Hille Ris Lambers (Homoptera: Aphididae). J. Bom. Nat. Hist. Soc. 88, 293-296. 
[96] Ghosh, A.K. and Raychaudhuri, D.N., A preliminary accounts of the bionomics and taxonomy of aphids from Assam. J. Bombay nat. Hist. Soc. 59, 238-253 (1962).

[97] Raha, S.K., Studies on the aphids (Homoptera, Insecta) of Nagaland, Ph. D. thesis, University of Calcutta, India, pp. 212 (1979).

[98] Raychaudhuri, D.N., Raha, S.K. and Raychaudhuri, D., Three new species of aphids (Homoptera: Aphididae) from Manipur and Nagaland. Entomon 2, 71- 75 (1977).

[99] Singh. T.K., Raychaudhuri, D., Raha, S.K. and Raychaudhuri, D.N., Hitherto unknown morphs of aphids (Homoptera: Aphididae) from Manipur and Nagaland, north east India. Entomon 5, 141-150 (1980).

[100]David, S.K., Aphids capable of infesting potato in India and their relationship to the crop in South India. J. South Indian Hort. 6, 67-74 (1958).

[101] Basu, A.N., Addition to the aphid of West Bengal. Sci. and Cult. 27, 456 (1961).

[102] Behura, B.K., Suppliment to aphids of India - a survey of published information. Prakruti - J. Utkal Univ. Sci. 3, 40-65 (1965).

[103] Saha, J.L., Poddar, S.C., Das, S.K., Agarwala, B.K. and Raychaudhuri, D., Studies on the aphid parasites (Hymenoptera: Aphidiidae) from Himachal Pradesh, India. Akitu, N.S. 44, 1-12 (1982).

[104]David, S.K., Rajasingh, S.G. and Narayanan, K., Notes on the taxonomy and other aspects of certain species of aphids in India. J. Bombay nat. Hist. Soc. 66, 508-512 (1968).

[105] Mall, N., Srivastava, P.N. and Singh, R., First record of host plants of aphids (Homoptera : Aphididae) from India. J. Aphidol. 24 (1-2), 85-86 (2010).

[106] Ghosh, A.K., A list of aphids (Homoptera, Aphididae) from India and adjacent countries. J. Bombay nat. Hist. Soc. 71, 201-225 (1974).

[107]Biswas, S., Chakrabarti, S., Basu, R.C, and Ghosh, A.K., New records of aphids (Insecta : Homoptera ) from Assam. Sci. and Cult. 35, 639-640 (1969).

[108] Ghosh, A.K., Banerjee, H. and Raychaudhuri, D.N., Aphids (Homoptera, Aphididae) collected from Kameng district, NEFA. Gaveshana 3, 10 (1971).

[109] Chakrabarti, S. Ghosh, A.K. and Raychaudhuri, D.N., New Records of Aphids (Insecta: Homoptera) from Uttar Pradesh, India. Sci. and Cult. 37, 247-248 (1971).

[110] Chowdhuri, A.N. Basu, R.C. and Raychaudhuri, D.N., A new species of Cavariella del Guercio and other newly recorded aphids (Homoptera, Aphididae) from Simla, Himachal Pradesh. Sci. and Cult. 35, 334 (1969).

[111] Ghosh, A.K., Chakrabarti, S., Chowdhuri, A.N. and Raychaudhuri, D.N., Aphids (Homoptera) of Himachal Pradesh, India. Orient. Ins. 3, 327-334 (1969).

[112] Stary, P. and Raychaudhuri. D.N., Aphid parasitoids (Hymenoptera : Aphidiidae) from northwestern India. Orient. Ins. 16, 297-304 (1982).

[113] Chakrabarti, S., Ghosh, A.K. and Chowdhuri, A.N., Aphids (Homoptera) of Himachal Pradesh, India-III. Oriental Ins. 4(4), 447-452 (1970).

[114] Chakrabarti, S., Ghosh, A.K. and Chowdhuri, A.N., Aphids (Homoptera: Aphididae) of Himachal Pradesh, India. Orient. Ins. 4, 447-452 (1970).

[115] Chakrabarti, S., Ghosh, A.K. and Raychaudhuri, D.N., Three new species of aphids (Homoptera : Aphididae) from North West Himalaya, India. Kontyu 39(4), 368-372 (1971).

[116] Chakrabarti, S., Ghosh, A.K. and Raychaudhuri, D.N., Some new aphids (Homoptera: Aphididae) from Himachal Pradesh, India. Orient. Ins. 8, 521-530 (1974).

[117] David, S.K., A taxonomic review of Macrosiphum in India. Orient. Ins. 9, 461-493 (1975).

[118] Raychaudhuri, D.N., Ghosh, M.R. and Basu, R.C., Subfamily : Aphidinae. In. Taxonomy of the aphids of North-east India and Bhutan, The Zoological Society, Calcutta, pp. 47- 278 (1980).

[119] Chakrabarti, S., Chowdhuri, A.N. and Raychaudhuri, D.N., Further records of aphids (Homoptera, Aphididae) from Himacha Pradesh, India. Sci. and Cult. 40, 461-462 (1974).

[120] Chowdhuri, A.N. Basu, R.C. Chakrabarti, S. and Raychaudhuri, D.N., A list of aphids from Simla including some new and a few others hitherto not known from India. Sci. and Cult. 34, 133-134 (1968). 
[121] Verma, K.D., A new genus, some species, a subspecies and some new records of aphids from N. W. India. Sci. and Cult. 35, 28-29 (1969).

[122] Verma, K.D., A new subspecies of Impatientinum impatiensae (Shinji) and the male of Protrama penecaeca Stroyan from northwest India (Homoptera: Aphididae). Bull. Ent. 10, 102103 (1969).

[123] Ghosh, A.K. Ghosh, M.R. and Raychaudhuri, D.N., Studies on the aphids (Homoptera: Aphididae) from eastern India. VII. New species and new records from West Bengal. Orient. Ins. 5, 209-222 (1971).

[124] Ghosh, A.K. and Raychaudhuri, D.N., Further records of aphids (Homoptera : Insecta) from NEFA. Sci. and Cult. 37, 483-484 (1971).

[125] Agarwala, B.K., Mondal, P.K. and Raychaudhuri, D.N., One new subgenus, three new species and one new subspecies of aphids (Homoptera) from Sikkim, northeast India. Entomon 7, 37-45 (1982).

[126] Hille Ris Lambers, D. and Basu, A.N., Some new or little known genera, subgenera, species and subspecies of Aphididae from India (Homoptera: Aphididae). Ent. Bericht. 26, 12-20 (1966).

[127] Remaudiere, G. and Remaudiere, M., Catalogue of the World's Aphididae. Instut National de la Recherche Agronomique, Paris, pp. 473 (1997).

[128] Chowdhuri, A.N., Basu, R.C., Chakrabarti, S. and Raychaudhuri, D.N., A list of aphids from Simla including some new and a few others hitherto not known from India. Sci. and Cult. 34, 133-134 (1968).

[129] Chowdhuri, A.N.., Basu, R.C., Chakrabarti, S. and Raychaudhuri, D.N., Aphids (Homoptera) of Himachal Pradesh, India. Orient. Ins. 3, 83-92 (1969).

[130] Ghosh, A.K., Basu, R.C. and Raychaudhuri, D.N., On a collection of aphids (Homoptera : Aphididae) from Bhutan with description of two new species. Kontyu, Tokyo 39, 120-125 (1971).

[131]Ghosh, A.K., Banerjee, H. and Raychaudhuri, D.N., Studies on the aphids (Homoptera: Aphididae) from eastern India. V. New species, new records on sexuals of some species of aphids from NEFA and Assam. Orient. Ins. 5, 103-110 (1971).

[132] Basu, A.N., New genera and species of aphids from the Darjeeling district, India (Homoptera, Aphididae). J. Linn. Soc. Zool. 45, 223-243 (1964).

[133] Chakrabarti, S. and Raychaudhuri, D.N., Hitherto unknown morphs of two species of aphids (Homoptera: Aphididae) described from India. Indian J. Entomol. 36, 128-131 (1974).

[134] Bhattacharya, D.K., Indumasonaphis chakrabartii, a new aphid (Homoptera : Aphididae) from Northeast India. Proc. zool. Soc., Calcutta 44, 127-130 (1991).

[135] Ghosh, A.K. and Raychaudhuri, D.N., Studies on the aphids (Homoptera, Aphididae) from eastern India. XIII. New species and further new records from Assam. Orient. Ins. 6, 371-386 (1972).

[136]Das, B.C. and Ghosh, L.K., Two new species of aphids (Homoptera: Aphididae) from Uttaranchal, India. J. Aphidol. 16, 73-76 (2002).

[137] Sathe, T.V. and Jadhav, B.V., Indian Pest Aphids. Daya Publishing House, New Delhi, pp. 211 (2008).

[138] Saha, Santanu and Chakrabarti, S., New records of aphids (Homoptera: Aphididae) from Garhwal range of Western Himalaya, India. J. Bom. nat. Hist. Soc. 85, 633-635 (1988).

[139] Chakrabarti, S., Saha, S. and Mandal, A.K., Longisiphoniella subterranea gen. et sp. nov. and hitherto unknown aphids (Homoptera : Aphididae) from Garhwal range of Western Himalaya. Proc. zool. Soc. Calcutta 37, 35-44 (1988).

[140]Chakrabarti, S. and Raychaudhuri, D.N., New and little known aphids (Homoptera : Aphididae) from Kumaon Himalaya, India. Entomon 3, 95-103 (1978).

[141]Ghosh, M.R., Basu, R.C. and Raychaudhuri, D.N., Studies on the aphids (Homoptera: Aphididae) from eastern India. XXXV. Three new genera and four new species from northeast India. Orient. Ins. 11, 579-586 (1977).

[142]Banerjee, H., Ghosh, A.K. and Raychaudhuri, D.N., On a collection of aphids (Homoptera, Aphididae) from Kullu valley, west Himalaya. Orient. Ins. 3, 255-264 (1969). 
[143] Nadda, G. and Joshi, S., First report of Aphid, Liosomaphis ornata Miyazaki, 1971 (Hemiptera: Aphididae) from India. Halteres 6, 51-55 (2015).

[144]Bhagat, R.C., New records and hosts of aphid parasitoids (Hymenoptera: Aphidiidae) from Kashmir, India. J. Bombay nat. Hist. Soc. 81, 93-98 (1984).

[145] Verma, K.D., Additions to the aphid fauna of north west India. Sci. and Cult. 31, 389 (1965).

[146] Bhalla, O.P., Addition to the aphid fauna of Himachal Pradesh. Himachal J. Agric. Res. 1, 51 $52(1971)$.

[147] Maity, S.P. and Chakrabarti, S., Aphids (Homoptera, Aphididae) of northwest India. III. Records of new aphids from Garhwal Himalaya. Sci. and Cult. 45, 160-162 (1979).

[148] Jadhav, V.B. and Sathe, T.V., Biodiversity of aphids (Order Hemiptera) from Satara district including western Ghat. In Biodiversity and Environment (Eds. Pandey, B.N. and Kulkarni, G.K.), APH Publishing Corporation, New Delhi, pp. 67-72 (2006).

[149] Banerjee, S.N. and Basu, A.N., Aphididae of West Bengal. Curr. Sci. 24: 61 (1955).

[150]Gupta, M.P., Reaction of ethiopean mustard, Brassica carinata Braun genotypes to mustard aphid Lipaphis erysimi (Kalt.). Indian J. Ecol. 30, 121-123 (2003).

[151] Sengupta, G.C., Das, J.N. and Behura, B.K., A preliminary account of the aphids of Orissa. Prakruti - J. Utkal Univ. Sci. 2, 33-39 (1962).

[152] Bakhetia, D.R.C., Labana, K.S., Sukhija, H.S. and Brar, K.S., Abst. Symp. 'Recent Trends in Aphidological Studies', Bhubaneswar, pp. 33-34 (1979).

[153] Devi, C.M., Singh, T.K. and Varathrajan, R.), Role of natural enemies in the management of Lipaphis erysimi (Kalt.) on Brassica juncea var. rugosa (Linn.). J. Biol. Cont., 16, 27-30 (2002.

[154]Nayak, M.R.C., Basu, M. and Raychaudhuri, D.N., Parasites and predators of aphids (Homoptera: Aphididae) from India. Pranikee 3, 7-14 (1982).

[155] Raychaudhuri, D.N. and Ghosh, A.K., A preliminary account of aphids of Rajasthan. Indian Agric. 3, 17-22 (1959).

[156] Ghosh, L.K., On a collection of aphids (Homoptera: Aphididae) from Rajasthan, India. Indian J. Sci. and Indust. (B) 4, 85-89 (1970).

[157] Ghosh, A.K. and Raychaudhuri, D.N., Aphids of Rajasthan. II. Indian Agric. 4, 228-229 (1962).

[158] Raychaudhuri, D.N. and Ghosh, A.K., A note on aphids of Calcutta and suberbs with special reference to newly recorded host plant families for some rare species. Curr. Sci. 27, 402 (1958).

[159] van der Goot, P., Zur Kenntnis der Blattläuse Javas. Contributions à la faune des Indes Néerlandaises 1(3), 1-301 (1917).

[160] Krishnamurthi, B., Aphididae of Mysore. I. J. Bombay nat. Hist. Soc. 33, 211-215 (1929).

[161]Bhagat R.C., Aphids (Insecta) of agricultural importance in J\&K state, India: a checklist and biodiversity. Internat. J. Food, Agric. and Vet. Sci. 2(3), 116-125 (2012).

[162] Rizvi, S.M.A. and Paul Khurana, S.M., Aphid fauna of economic crop plants in Gorakhpur. Sci. and Cult. 36, 49 (1970).

[163] Chakrabarti, S. and Banerjee, P.K., A review of the genus Longicaudus (Homoptera: Aphididae) with descriptions of two new species from India. Orient. Ins. 25, 199-210 (1991).

[164]Chakrabarti, S. and Banerjee, P.K., Host-alternation in Aphids from Western and Northwest Himalaya, India. Proceedings of Critical Issues in Aphid Biology, pp. 43-53 (1993).

[165] Ghosh, A.K., Ghosh, M.R. and Raychaudhuri, D. N., Studies on aphids (Homoptera: Aphididae) from eastern India. II. Some new species and new records from north Bengal. Orient. Ins. 4, 193-203 (1970).

[166] Ghosh, A.K., Fern infesting aphids (Homoptera: Aphididae) in India. Indian. J. Hort. 31, 104 109 (1974).

[167] Ghosh, L.K. and Basu, R.C., Insecta, Hemiptera, Aphididae. In Fauna of West Bengal. State Fauna Series, Zool. Surv. India 3, 125-318 (1997).

[168] Ghosh, L.K., Additions to the aphid fauna of Bihar with the first record of an aphid sexuale (Homoptera: Aphididae). J. Bombay nat. Hist. Soc. 73, 416-418 (1977).

[169] Ghosh, M.R., Ghosh, A.K., and Raychaudhuri, D.N., Studies on aphids (Homoptera, Aphididae) from eastern India. Proc. zool. Soc. Calcutta, 24, 163-168 (1971). 
[170]Basu, R.C., Ghosh, A.K. and Raychaudhuri, D.N., Studies on the aphids (Homoptera, Aphididae) from eastern India. VIII. A new genus and records of aphids from Assam. Sci. and Cult. 40, 41-43 (1974).

[171]David, S.K. and Narayanan, K., Three new species of aphids from southwestern Himalayas in India. Bull. Ent. 9, 99-103 (1968).

[172] Ghosh, M.R., Ghosh, A.K. and Raychaudhuri, D.N., Studies on the aphids (Homoptera: Aphididae) from eastern India. VI New records of aphids from Sikkim. Proc. zool. Soc. Calcutta 24, 47-51 (1971).

[173] Raha, S.K. and Raychaudhuri, D.N., A new species of Macromyzus Takahashi from West Bengal. Entomon 3, 111-113 (1978).

[174]Basu, R.C. and Raychaudhuri, D.N., Studies on the aphids (Homoptera: Aphididae) from eastern India. XXIX. The genus Macrosiphoniella. Orient. Ins. 10, 295-306 (1976).

[175]Ghosh, A.K., Basu, R.C. and Raychaudhuri, D.N., A new genus and seven new species of aphids (Homoptera) from India. Orient. Ins. 3, 245-254 (1969).

[176]Medda, P.K. and Chakrabarti, S., On three undescribed morphs of Macrosiphine aphids (Homoptera: Aphididae) from western Himalaya. India. Proc. zool. Soc. Calcutta 51, 57-59 (1998).

[176a] Joshi, S. and Sangma, R.H.C. Natural enemies associated with aphids and coccids from Sikkim, India. J.Biol. Cont. 29(1), 3-7 (2015).

[177] Saha, S. and Chakrabarti, S., New species, hitherto less known and unknown morphs of aphids (Homoptera : Aphididae) from India. Proc. zool. Soc. Calcutta 38, 21-28 (1988).

[178] Verma, K.D. and Mathur, A.C., First record of two species and males of Lipaphis pseudobrassicae Davis and Protrama panecaceae Stroyan (Homoptera: Aphididae) from India. Indian J. Entomol. 28, 277-278 (1966).

[179] Khuda-Bukhsh, A.R. and Pal, N.B., Cytogenetical studies on aphids (Homoptera: Aphididae) from India. III. Karyomorphology of fifteen species belonging to the tribe Macrosiphini. Entomon 11, 141-147 (1986).

[180] Raychaudhuri, D.N., Ghosh, D., Raychaudhuri, D. and Agarwala, B.K., Studies on the aphids (Homoptera: Aphididae) from south India. I. Ins. Matsum. n. s. 23, 1-20 (1981).

[181] Raychaudhuri, D.N., Dutta, S., Agarwala, B.K., Raychaudhuri, D. and Raha, S.K., Some parasites and predators of aphids from northeast India and Bhutan. Entomon 3, 91-94 (1978).

[182] Das, B.C., The Aphididae of Lahore. Mem. Indian Mus. 6(4), 135-274 (1918).

[183] Agarwala, B.K. and Raychaudhuri, D.N., Parasites and predastors of aphids in Sikkim and Manipur (northeast India). III. Entomon 5, 39-42 (1980).

[184] Ghosh, L.K., On a collection of aphids (Homoptera, Aphididae) from Himachal Pradesh, India. Orient. Ins. 6, 169-178 (1972).

[185] Agarwala, B.K. and Raychaudhuri, D.N., Two new species of aphids (Homoptera, Aphididae) from Sikkim, northeast India. Entomon 2, 77-80 (1977).

[186] Krishnamurthi, B., Aphididae of Mysore. III. Indian J. Ent. 10, 51-53 (1948).

[187]Bindra, O.S. and Sekhon, S.S., New records of aphids from Punajb and Kulu valley. Bull. Ent. 10, 103-104 (1969).

[188] David, S.K., Notes on South Indian Aphids. Indian J. Ent. 18, 1-9 (1956).

[189] David, S.K., A new genus and three new species of aphids from India. Indian J. Ent. 20, 175180 (1958).

[190] Chakrabarti, S. and Ghosh, A.K., On the rose infesting aphids (Homoptera: Aphididae) in India. Indian J. Hort. 27, 226-232 (1970).

[191]David, S.K., Some rare Indian aphids. J. Bombay nat. Hist. Soc. 55, 110-116 (1958).

[192] Shuja Uddin., Two new species of the genus Toxares Westwood (Aphidiidae: Hymenoptera) from India with note on the genus. Indian J. Entomol., 36, 268-274 (1974).

[193]Lefroy, H.M and Howlett, F.M., Indian Insect Life. A manual of the insects of the plains (Tropical India), W. Thacker and Co. London, pp. $743-748$ (1909). 
[194] Agrawal, R. and Singh, R., New host records of aphids (Homoptera: Aphididae) in northeastern Uttar Pradesh. J. Aphidol. 19(1-2), 109-111 (2005).

[195]Despande, V.G., A preliminary account of the Aphididae of Poona. J. Bombay nat. Hist. Soc. 39, 740-744 (1938).

[196] Chakrabarti, S. and Raychaudhuri, D.N., New record of aphids (Homoptera : Aphididae) from Nepal. Curr. Sci. 41(23), 858-859 (1972).

[197] Basu, R.C. and Raychaudhuri, D.N., Studies on the aphids (Homoptera, Aphididae) from eastern India. XXXI. The genus Capitophorus. Orient. Ins. 10, 567-572 (1976).

[198] Saha, B.K., Samanta, A.K., Pramanik, D.R. and Raychaudhuri, D., Some new and hitherto unknown aphids (Homoptera : Aphididae) from India. Orient. Zool. 3, 79-89 (1983).

[199] Ghosh, A.K. and Raychaudhuri, D.N., Aphids (insecta: Homoptera) of Sikkim. Proc. zool. Soc. Calcutta 21, 179-195 (1968).

[200] Chowdhuri, A.N. Ghosh, A.K. Banerjee, H. and Raychaudhuri, D.N., A few sexual forms and seven newly recorded species of aphids (Aphididae, Homoptera) from Simla. Sci. and Cult. 36, 550 (1970).

[201] Ghosh, A.K., Taxonomical notes on some species of Indian aphids (Homoptera : Aphididae). Orient. Ins. 7, 347-350 (1973).

[202] Ghosh, L.K., Metopolophium (Metopolophium nov. subgen.) darjeelingensis nov. sp. (Homoptera: Aphididae) from West Bengal, India. Bull. Ent. 11(2), 116-117 (1970).

[203] David, S.K. Narayanan, K. and Rajasingh, S.G., A new genus and four new species of aphids (Homoptera) from India. Oriental Ins. 5, 557-570 (1971).

[204] Maity, S.P. Bhattacharya, D.K. and Chakrabarti, S., Aphids (Homoptera: Aphididae) of northwest India. V. New records of aphids from Garhwal Himalaya. Sci. and Cult. 46, 311-312 (1980).

[205]Medda, P.K. and Chakrabarti, S., Hitherto unknown morphs of Eumyzus pruni and Metopolophium simlaense (Homoptera : Aphididae) from Western Himalaya, India. Entomon 14(1and2), 29-32 (1989).

[206] Agarwala, B.K. and Mahapatra, S.K., Description of hitherto unknown sexual morphs of five species of Aphididae (Homoptera) from India. Orient. Ins. 24, 237-246 (1990).

[207] David, S.K. and Hameed, S.F., One new species and two new records of aphids (Homoptera, Aphididae) from Lahaul in northwestern Himalaya. Orient. Ins. 9, 213-219 (1975).

[208] Mondal, P.K., Basu, R.C. and Raychaudhuri, D.N., Studies on the aphids (Homoptera : Aphididae) from eastern India. XXX. The Genus Toxoptera. Orient. Ins. 10, 533-540 (1976).

[209] Bhagat, R.C., On two new parasitoids of genus Trioxys (Aphidiidae: Hymenoptera) from Kashmir, India. Entomon 7, 321-324 (1982).

[210]Das, B.C., Bhattacharya, A., Ghosh, L.K. and Goswami, A., A new species of aphid (Homoptera: Aphididae) from Sikkim, India. J. Aphidol. 19, 37-40 (2005).

[211] Bhanotar, R.K. and Ghosh, L.K., Micromyzus judenkoi Carver (Homoptera : Aphididae ), a new record for India. Sci. and Cult. 35, 72 (1969).

[212] Ghosh, L.K., Description of new species of Micromyzodium David (Homoptera) from the western Himalaya. Orient. Ins. 4, 435-439 (1970).

[213] Agarwala, B.K. and Raychaudhuri, D.N., An account of aphids (Homoptera : Aphididae) infesting the important economic plants in Sikkim. Indian Agric. 25, 101-107 (1981).

[214] Ghosh, M.R., Basu, R.C. and Raychaudhuri, D.N., Studies on aphids (Homoptera, Aphididae) from eastern India. XXVIII. Acutosiphon, Akkaia and Vesiculaphis. Orient. Ins. 10, 267-276 (1976).

[215] David, S.K. Rajasingh, S.G. and Narayanan, K., The myzaphidines (Homoptera) of India with description of three new species. Orient. Ins. 4, 395-406 (1970).

[216] Basu, R.C. and Raychaudhuri, D.N., Studies on the aphids (Homoptera: Aphididae) from eastern India. XXV. The genus Myzus with five new species from eastern India. Orient. Ins. 10, 93-112 (1976).

[217] Agarwala, B.K. and Ghosh, A.K., Monograph on oriental Aphidoidea. Key to the genera and synoptic list. Mem. zool. Surv. India 16,1-118 (1985). 
[218]Bhattacharya, D.K. and Chakrabarti, S., Stratigraphic distribution and host association of Aphidinae aphids (Homoptera, Aphididae) of Garhwal range of Western Himalaya. J. Aphidol. 1, 64-69 (1987).

[219] Akhtar, M.S., Dey, D., and Usmani, M.K., A catalogue of aphid parasitoids (Hymenoptera: Braconidae: Aphidiinae) from India. Insecta Mundi 0151, 1-31 (2011).

[220] Ghosh, A.K., New species and new records of aphids (Homoptera : Aphididae) from Northeast India. Orient. Ins. 8, 161-175 (1974).

[221] Khuda-Bukhsh, A.R. and Pal, N.B., Cytogenetical studies on aphids (Homoptera: Aphididae) from India. II. Karyomorphology of five species of Myzus. Entomon, 11, 135-140 (1986).

[222]Basu, A.N. and Ganguli, B., A note on the transmission of 'foorkey disease' of large cardamom by the aphid, Micromyzus kalimpongensis Basu. Indian Phytopathol. 21, 127 (1968).

[223] Ghosh, A.K., New species, undescribed morphs and new records of aphids (Homoptera: Aphididae) from northeast India. Orient. Ins. 10, 39-49 (1976).

[224] Subhrani, S, Singh, P.M. and Singh, T.K., Biodiversity of aphidiine parasitoids (Hymenoptera: Braconidae) on certain crops in Manipur. J. Aphidol. 20(1), 1-4 (2006).

[225] David, S.K., Narayanan, K. and Rajasingh, S.G., A new genus and four new species of aphids (Homoptera: Aphididae) from India. Orient. Ins. 5, 557-570 (1971).

[226] Ghosh, A.K. and Agarwala, B.K., Weed hosts of major aphid (Homoptera : Insecta) pests in India region. Indian Agric. 24, 101-107 (1980).

[227] Raychaudhuri, D.N., Ghosh, M.R., Banerjee, M. and Ghosh, A.K., Studies on the aphids (Homoptera : Aphididae) from eastern India. XIV. One new genus, two new sub genera and fifteen new species of Greenideini. Kontyu 41, 53-73 (1973).

[228] Ghosh, A.K. and Raychaudhuri, D.N., A preliminary account of bionomics and taxonomy of aphids from assam. II. J. Asiat. Soc. Bengal 4, 101-113 (1962).

[229] Ghosh, A.K. and Raychaudhuri, D.N., Additions to the aphid fauna of Assam. Sci. and Cult. 29, 104 (1963).

[230]Raha, S.K. and Raychaudhuri, D.N., Studies on the aphids (Homoptera, Aphididae) of Nagaland. Entomon, 6, 317-323 (1981).

[231]Das, B.C. and Chakrabarti, S., Seasonal occurrence of Aphidius matricariae Haliday (Aphidiidae, Hymenoptera) in Garhwal range of northwest Himalaya. Indian J. Ent. 50, 388-389 (1988).

[232] Raychaudhuri, D., Raychaudhuri, D.N. and Singh, T.K.), Redescription of Shiavaphis celti (oviparae) and Myzus varians (Homoptera : Aphididae) hitherto unknown from India. Sci. and Cult. 47, 171 - 172 (1981.

[233] Singh, T.K. and Raychaudhuri, D., Aphids and their coccinelid predators of fruit trees in Manipur, northeast India. J. Aphidol. 1, 78-79 (1987).

[234]Debnath, M., Medda and Chakrabarti. . Diversity of aphids (Hemiptera: Aphididae) on the plants of Brassicaceae (Cruciferae) in India. Hexapoda 17(2), 101-106 (2010).

\section{AUTHORS' BIOGRAPHY}

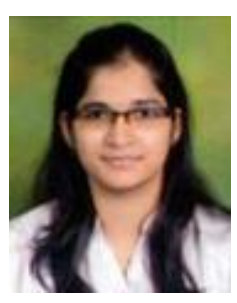

Ms. Garima Singh, is a Ph.D. Student in the Department of Zoology, Rajasthan University, Jaipur and doing research work on the factors affecting progeny sex ratio of an aphid parasitoid, Lipolexis oregmae (Gahan) (Hymenoptera: Braconidae, Aphidiinae). She has published three review articles. She has compiled the food plants of black bean aphid, Aphis craccivora Koch, cotton aphid, Aphis gossypii Glover, green peach aphid, Myzus persicae (Sulzer) and spirea aphid, Aphis spiraecola Patch recorded in India.

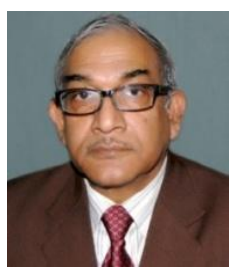

Dr. Rajendra Singh, is Professor and Head of the Department of Zoology, Deen Dayal Upadhyaya University of Gorakhpur and has 40 years of experience in the fields of bioecology and biosystematics of aphids and their parasitoids and predators, published more than 200 research articles of international repute, authored 6 text/reference books, supervised one D.Sc. and $25 \mathrm{Ph}$. D. students and completed 12 research projects. He is fellow/life member of several scientific organizations/ societies, Chief Editor of J. Aphidol. and is on the editorial/reviewer panel of several International Journals. Prof. Singh also served in the university as Pro-Vice Chancellor. 